\title{
A UNIFIED APPROACH TO XVA WITH CSA DISCOUNTING AND INITIAL MARGIN
}

\author{
FRANCESCA BIAGINI, ALESSANDRO GNOATTO, AND IMMACOLATA OLIVA
}

March 4, 2021

\begin{abstract}
In this paper we extend the existing literature on xVA along three directions. First, we enhance current BSDE-based xVA frameworks to include initial margin in presence of defaults. Next, we solve the consistency problem that arises when the front-office desk of the bank uses trade-specific discount curves (CSA discounting) which differ from the discount rate adopted by the xVA desk. Finally, we clarify the impact of aggregation of several sub-portfolios of trades on the xVA-valuation of the resulting global portfolio and study related non-linearity effects.
\end{abstract}

\section{INTRODUCTION}

As a consequence of the 2007-2009 financial crisis, academics and practitioners are revisiting the valuation of financial products in several aspects. In particular, the value of a product should account for the possibility of default of any agent involved in the transaction. Financial regulations, such as Basel III/IV and Emir, are also driving the methodological development.

All these issues are represented at the level of valuation equations by introducing value adjustments (xVA), which are further terms to be added or subtracted to an idealized reference price, computed in the absence of the aforementioned frictions, in order to obtain the final value of the transaction.

In this paper we aim to provide a unified and comprehensive framework for pricing counterparty risk with $\mathrm{xVA}$ and assessing funding costs by using BSDEs techniques in a market described by diffusions. We then consider the consistency problem between xVA pricing equations and the CSA discounting rules, which originates from the quoting mechanism of market standard instruments, and solve it by relying on an invariance property of linear BSDEs. Furthermore, we extend our approach to include pricing of a global portfolio possibly stratified in an aggregation of several subsets of claims, and study non-linearity effects due to incremental xVA charges.

In order to better specify our contribution, we first shortly recall the literature on xVA. Since the research on $\mathrm{xVA}$ is very wide, our overview is far from being exhaustive. The first contribution on the subject is Duffie and Huang (1996). Before the 2007-2009 financial crisis, the works of Brigo and Masetti (2005) and Cherubini (2005) analyze the concept of credit valuation adjustment (CVA) for the first time. The possibility of default of both counterparties involved in the transaction, represented by the introduction of the debt valuation adjustment (DVA), is investigated, among others, in Brigo et al. (2011) and Brigo et al. (2014).

Apart from the issue of default risk, another important source of concern for practitioners and academics is represented by funding costs.

A parallel stream of literature emerged during and after the financial crisis, to generalize valuation equations to account for features such as the presence of collateralization agreements. In a BlackScholes economy, Piterbarg (2010) provides valuation formulas in presence or absence of collateral 
agreements. Piterbarg (2012) generalizes the issue in a multi-currency economy, see also Fujii et al. (2010), Fujii et al. (2011), and Gnoatto and Seiffert (2020). The funding valuation adjustment (FVA) under several alternative assumptions on the so called Credit Support Annex (CSA) is derived in Pallavicini et al. (2011), while Brigo and Pallavicini (2014) also discusses the role of central counterparties in the context of funding costs. A general approach to funding issues in a semimartingale setting is provided by Bielecki and Rutkowski (2015).

Both funding and default risk need to be unified in a unique pricing framework. Contributions in this sense can be found in Brigo et al. (2018) by means of the so-called discounting approach. In a series of papers, Burgard and Kjaer generalize the classical Black-Scholes replication approach to include many effects, see Burgard and Kjaer (2011b) and Burgard and Kjaer (2013). A more general BSDE approach is provided by Crépey (2015a), Crépey (2015b), Bichuch et al. (2018a) and Bichuch et al. (2018b). The equivalence between the discounting and the BSDE-based replication approaches is demonstrated in Brigo et al. (2018).

The importance of $\mathrm{xVA}$ is reflected by the increasing number of monographs on the subject, see e.g. Brigo et al. (2013). An advanced BSDE-based treatment is provided by Crépey et al. (2014). A detailed analysis of how to construct large hybrid models for counterparty risk simulations are provided in Green (2015), Lichters et al. (2015) and Sokol (2014), while Gregory (2015) provides an accessible introduction to most aspects of the topic.

The present paper contributes to the literature on $\mathrm{xVA}$ along the following directions: on the theoretical side, we provide a unifying treatment of different aspects of $\mathrm{xVA}$ that have been separately treated by different authors in a comprehensive approach; on the other hand, we provide a rigorous mathematical treatment of practical issues arising in the context of $\mathrm{xVA}$ calculation and management in current market practice.

More precisely, we propose an xVA framework using BSDEs techniques in a market described by diffusions. Our BSDEs, introduced under a progressively enlarged filtration $\mathbb{G}$, are specified up to a random time horizon, given by the minimum between the default time of the counterparty, the default time of the bank, and the maturity of the contract. For the sake of simplicity, we first discuss the well posedness of the BSDE for a portfolio including a contingent claim. Our approach unifies several contributions from the existing literature. First, we include initial margins in the BSDE driver as in Agarwal et al. (2018), but we extend their setting by including defaults. Moreover, we consider different discounting rules without resorting to measure changes as in Bichuch et al. (2018a). Finally, we provide a price decomposition in terms of a clean value and an adjustment process, by considering the associated pre-default BSDE with respect to the reference filtration $\mathbb{F}$ along the lines of Crépey (2015a), Crépey (2015b) and Bielecki and Rutkowski (2015).

Concerning discounting rules, it is nowadays accepted that the trading activity is funded by resorting on different sources of liquidity. More precisely, the existence of different funding curves leads to the consistency problem between xVA pricing equations and the so-called CSA discounting rules. The latter originates from the quoting mechanism of market standard instruments. Such instruments are quoted under the assumption that they are perfectly collateralized. Since a perfectly collateralized transaction is funded by the collateral provider, the discounting rate applied to evaluate market instruments is given by a collateral rate, which typically corresponds to an overnight interest rate. The presence of multiple assumptions on the collateral rate implies the co-existence of quotes with different discounting rates, which are in general at odds with the discounting rate dictated by the $\mathrm{xVA}$ pricing BSDE. A further contribution of our work consists in a solution to the consistency issue by relying on an invariance property of linear BSDEs. This is realized by introducing a further 
value adjustment term, called Discounting Value Adjustment (DiscVA), representing the clean price adjustment compared to the one officially accepted by the front-office desk.

Moreover, the counterparty could be economically related to other entities, a typical example being given by a parent-subsidiary relation or in general by the existence of dedicated sub-portfolios. Such a scenario results in a multi-layer hierarchical structure in the resulting global portfolio, which also takes into account the potentially different funding policies implemented by each of the aforementioned financial entities. We study the impact of such a stratified structure on the formulation of pricing equations. In realistic situations, the global portfolio of positions between the bank and the counterparty is in general an aggregation of several subsets of claims, whose structure is dictated by legal agreements in force between the two agents. We allow for the presence of multiple agreements for the exchange of margins (margin sets) and multiple netting sets. We adapt our xVA framework to arbitrary configurations of aggregation levels, while preserving the well posedness of the underlying BSDE.

Finally, we present incremental xVA charges for new potential trades under the proposed xVA framework: given the presence of portfolio effects in the computation of value adjustments, and given an existing portfolio of $K$ trades, the xVA charge for a new potential $(K+1)$-th trade is computed as the difference of the $\mathrm{xVAs}$ of two portfolios. More precisely, this corresponds to the difference between the $\mathrm{xVA}$ of the extended portfolio, consisting of $(K+1)$ trades, and the $\mathrm{xVA}$ charge of the base portfolio of $K$ trades. In this way we are able to describe non-linearity of portfolio-wide valuation. Existing discussions concerning the impact of the incremental cash flow of a trade on the balance sheet of the dealer can be found in Burgard and Kjaer (2013), Castagna (2013), Castagna (2014).

Given our focus on discounting and aggregation levels, in this paper we do not discuss capital valuation adjustment (KVA). The issue is treated in recent papers such as Albanese and Crépey (2017), Albanese et al. (2016) and Albanese et al. (2017), see also the ongoing discussion in Andersen et al. (2019). This is beyond the scope of the present paper and leave it for future research.

The paper is organized as follows. In Section 2 we formalize in mathematical terms the main financial concepts related to the xVA framework. Section 3 first describes the results related to the xVA evaluation when only one transaction is taken into account, extending the analysis to CSA discounting. Furthermore, we study the xVA framework when the portfolio includes several contracts and investigate the role played by non-linearity. Section 4 provides an example and some numerical results illustrating the results of Section 3 . In Appendix A we gather some results from the literature used to derive the main results.

\section{The FINANCIAL SETTING}

We fix a time horizon $T<\infty$ for the trading activity. We consider two agents named the bank (B) and the counterparty $(\mathrm{C})$. Unless otherwise stated, throughout the paper we assume the bank's perspective and refer to the bank as the hedger. All processes are modeled over a probability space $(\Omega, \mathcal{G}, \mathbb{P})$. Let $W^{\mathbb{P}}=\left(W_{t}^{\mathbb{P}}\right)_{t \in[0, T]}$ be a $d$-dimensional Brownian motion on $(\Omega, \mathcal{G}, \mathbb{P})$ with associated natural filtration $\mathbb{F}$.

We denote by $\tau^{B}$, resp. by $\tau^{C}$, the time of default of the bank, resp. of the counterparty and by $H_{t}^{j}:=1_{\left\{\tau^{j} \leq t\right\}}, j \in\{B, C\}$, the associated jump process.

Default times $\tau^{B}$ and $\tau^{C}$ are assumed to be exponentially distributed random variables with timedependent intensity

$$
\Gamma_{t}^{j}=\int_{0}^{t} \lambda_{s}^{j, \mathbb{P}} d s, j \in\{B, C\}, t \in[0, T]
$$


where $\lambda^{j}$ are non-negative bounded $\mathbb{F}$-adapted processes. Let $\mathbb{H}^{j}=\left(\mathcal{H}_{t}^{j}\right)_{t \in[0, T]}, j \in\{B, C\}$, be the natural filtration of $H^{B}, H^{C}$, respectively. On $(\Omega, \mathcal{G}, \mathbb{P})$ we consider the filtration $\mathbb{G}=\mathbb{F} \vee \mathbb{H}$. All filtrations are required to satisfy the usual hypotheses of completeness and right-continuity.

In the present paper we will extensively make use of the so called Immersion Hypothesis.

Hypothesis 2.1. Any local $(\mathbb{F}, \mathbb{P})$-martingale is a local $(\mathbb{G}, \mathbb{P})$-martingale.

Note that, by Hypothesis 2.1. $W^{\mathbb{P}}$ is also a $\mathbb{G}$-Brownian motion. By Bielecki and Rutkowski 2004 , Corollary 5.2.4) we obtain that the predictable representation property holds in $\mathbb{G}$ with respect to $W, M^{B}, M^{C}$, where

for $j \in\{B, C\}$.

$$
M_{t}^{j}:=H_{t}^{j}-\int_{0}^{t \wedge \tau^{j}} \lambda_{u}^{j} d u, t \in[0 . T]
$$

From now on, we set

$$
\tau:=\tau^{B} \wedge \tau^{C} \wedge T
$$

2.1. Basic traded assets. We now introduce the market model. For $d \geq 1$, we denote by $S^{i}$, $i=1, \ldots, d$ the ex-dividend price (i.e. the price) of risky assets with associated cumulative dividend processes $D^{i}$. All $S^{i}$ are assumed to be càdlàg $\mathbb{F}$-semimartingales, while the cumulative dividend streams $D^{i}$ are $\mathbb{F}$-adapted processes of finite variation with $D_{0}^{i}=0$.

We consider the following coefficient functions:

$$
\begin{aligned}
& \mu:\left(\mathbb{R}_{+} \times \mathbb{R}^{d}, \mathcal{B}\left(\mathbb{R}_{+} \times \mathbb{R}^{d}\right)\right) \mapsto\left(\mathbb{R}^{d}, \mathcal{B}\left(\mathbb{R}^{d}\right)\right), \\
& \sigma:\left(\mathbb{R}_{+} \times \mathbb{R}^{d}, \mathcal{B}\left(\mathbb{R}_{+} \times \mathbb{R}^{d}\right)\right) \mapsto\left(\mathbb{R}^{d \times d}, \mathcal{B}\left(\mathbb{R}^{d \times d}\right)\right), \\
& \kappa:\left(\mathbb{R}_{+} \times \mathbb{R}^{d}, \mathcal{B}\left(\mathbb{R}_{+} \times \mathbb{R}^{d}\right)\right) \mapsto\left(\mathbb{R}_{+}^{d}, \mathcal{B}\left(\mathbb{R}^{d}\right)\right),
\end{aligned}
$$

which are assumed to satisfy standard conditions ensuring existence and uniqueness of strong solutions of SDEs driven by the Brownian motion $W^{\mathbb{P}}$. The matrix process $\sigma$ is invertible at every point in time. We assume that

$$
\begin{cases}d S_{t} & =\mu\left(t, S_{t}\right) d t+\sigma\left(t, S_{t}\right) d W_{t}^{\mathbb{P}} \\ S_{0} & =s_{0} \in \mathbb{R}^{d}\end{cases}
$$

on $[0, T]$. Note that we are not postulating that the processes $S^{i}$ are positive. The dividend processes $D^{i}$ are specified via

$$
\left(D_{t}^{1}, \ldots, D_{t}^{d}\right)^{\top}=\int_{0}^{t} \kappa\left(u, S_{u}\right) d u, t \in[0, T]
$$

for $\kappa$ given in 2.2 such that $\int_{0}^{T}\left|\kappa\left(u, S_{u}\right)\right| d u<\infty \mathbb{P}$-a.s.

The cumulative dividend price associated to the $i$-th asset is given by

$$
S_{t}^{i, c l d}:=S_{t}^{i}+B_{t}^{i} \int_{(0, t]} \frac{d D_{u}^{i}}{B_{u}^{i}}, \quad i=1, \ldots d, t \in[0, T] .
$$

We assume the existence of an indexed family of cash accounts $\left(B^{x}\right)_{x \in \mathcal{I}}$, where the stochastic process $r^{x}:=\left(r_{t}^{x}\right)_{t \geq 0}$ is bounded and $\mathbb{F}$-adapted for all $x \in \mathcal{I}$. The set of indices $\mathcal{I}$ embodies the type of agreement the counterparties establish in order to mitigate the counterparty credit risk. We will specify the characteristics of the aforementioned indices later on. 
All cash accounts, with unitary value at time 0 , are assumed to be strictly positive continuous processes of finite variation of the form

$$
B_{t}^{x}:=\exp \left\{\int_{0}^{t} r_{s}^{x} d s\right\}, t \in[0, T] .
$$

We introduce two risky bonds with maturity $T^{\star} \leq T$ and rate of return $\bar{r}^{j}+\lambda^{j}, j \in\{B, C\}$, issued by the bank and the counterparty, respectively, with dynamics

$$
d P_{t}^{j}=\left(\bar{r}_{t}^{j}+\lambda_{t}^{j}\right) P_{t}^{j} d t-P_{t-}^{j} d H_{t}^{j}, j \in\{B, C\} .
$$

The payment stream of a financial contract is represented by an $\mathbb{F}$-adapted càdlàg process of finite variation $A=\left(A_{t}\right)_{t \in[0, T]}$, as in Crépey (2015b). We use the notation $\Delta A_{t}:=A_{t}-A_{t-}$ for the jumps of $A$. To include the presence of default events, we define the process $\bar{A}=\left(\bar{A}_{t}\right)_{t \in[0, T]}$ by setting

$$
\bar{A}_{t}:=1_{\{t<\tau\}} A_{t}+1_{\{t \geq \tau\}} A_{\tau-} .
$$

Note that $A_{\tau-}$ represents the last payment before default, see also Brigo and Morini (2018).

2.2. Repo-trading and collateralization. In line with the existing literature, we assume that the trading activity on the risky assets is collateralized. This means that borrowing and lending activities related to risky securities are financed via security lending or repo market, see Bichuch et al. (2018a) Since transactions on the repo market are collateralized by the risky assets, repo rates are lower than unsecured funding rates. As argued in Crépey (2015a), assuming that all assets are traded via repo markets is not restrictive. In case the transactions are fully collateralized, this translates in the following equality

$$
\xi_{t}^{i} S_{t}^{i}+\psi_{t}^{i} B_{t}^{i}=0, i=1 \ldots, d, t \in[0, T],
$$

where $B^{1}, \ldots, B^{d}$ are the cash accounts associated to the risky assets $S^{1}, \ldots, S^{d}$.

It is worth noting that $\xi_{t}^{i}, i=1, \ldots, d$, may be either positive or negative. Here $\xi_{t}^{i}>0$ means that we are in a long position, which has to be financed by collateralization. On the other hand, $\xi_{t}^{i}<0$ implies that the $i$-th asset is shorted, so that the whole amount of collateral is deposited in the riskless asset. Condition (2.9) plays an important role in precluding trivial arbitrage opportunities among different cash accounts.

Within the bank, the trading desk borrows and lends money from/to the treasury desk. Borrowing and lending rates are allowed to differ, hence we denote by $r^{f, b}, r^{f, l}$ the rate at which the trading desk borrows from and lends to the treasury desk, respectively. In the same notation as in (2.6), we introduce the associated cash accounts $B^{f, b}, B^{f, l}$. This means that if the position of the trading desk is negative, i.e. $\psi^{f}=\psi^{f, b}<0$, the trading desk borrows from the treasury desk at the rate $r^{f, b}$. Conversely, if the position of the trading desk is positive, i.e. $\psi^{f}=\psi^{f, l}>0$, the trading desk lends money to the treasury desk with remuneration $r^{f, l}$. Since simultaneously borrowing and lending from the treasury desk is precluded, we set

$$
\psi_{t}^{f, l} \psi_{t}^{f, b}=0, \text { for all } t \in[0, T] .
$$

For what concerns financial contracts, collateralization is a method to minimize losses due to default of the counterparty by using margins. In the financial jargon, a margin represents an economic value, 
either in the form of cash or risky securities, exchanged between the counterparties of a financial transaction, in order to reduce their risk exposures. In line with the current market practice, we distinguish between initial margin and collateral (or variation margin), that we present in the following.

2.2.1. Variation margin. A collateral is posted between the bank and the counterparty to mitigate counterparty risk. The collateral process $C=\left(C_{t}\right)_{t \in[0, T]}$ is assumed to be $\mathbb{G}$-adapted. We follow the convention of Bichuch et al. (2018a) and Crépey (2015a):

- If $C_{t}>0$, we say that the bank is the collateral provider. It means that the counterparty measures a positive exposure towards the bank, so it is a potential lender to the bank, hence the bank provides/lends collateral to reduce its exposure.

- If $C_{t}<0$, we say that the bank is the collateral taker. It means that the bank measures a positive exposure towards the counterparty, so it is a potential lender to the counterparty, hence the counterparty provides/lends collateral to reduce its exposure.

Let $\mathcal{V}=\left(\mathcal{V}_{t}\right)_{t \in[0, T]}$ be a generic $\mathbb{G}$-adapted process, representing either the value of the trade including counterparty risk and funding adjustments or the clean value process, as it will be clarified later on. We assume that $C_{t}:=f\left(\mathcal{V}_{t}\right), t \in[0, T]$, where $f: \mathbb{R} \rightarrow \mathbb{R}$ is a Lipschitz function. This assumption allows to cover realistic collateral specifications, see e.g. Lichters et al. (2015) and Ballotta et al. (2019).

If there is a collateral agreement (or a multitude of agreements) between the bank and the counterparty, in evaluating portfolio dynamics we need to make a distinction between the value of the portfolio and the wealth of the bank, the two concepts being distinguished since the bank is not the legal owner of the collateral (prior to default).

In this paper collateral is always posted in the form of cash, in line with standard current market practice. Moreover, we assume rehypothecation, meaning that the holder of collateral can use the cash to finance her trading activity. This is the opposite of segregation, where the received cash collateral must be kept in a separate account and can not be used to finance the purchase of assets.

We associate the following interest rates to the collateral account:

- $r^{c, l}$ with account $B^{c, l}$, representing the rate on the collateral amount received by the bank who posted collateral to the counterparty.

- $r^{c, b}$ with account $B^{c, b}$, representing the rate on the collateral amount paid by the bank who received collateral from the counterparty.

We simply set $r^{c}=r^{c, l}=r^{c, b}$ in case there is no bid-offer spread in the collateral rate. Possible choices for the collateral rate are e.g. EONIA for EUR trades, Fed Fund for USD and SONIA for GBP trades. Such rates are overnight rates with a negligible embedded risk component. The choice of such approximately risk-free rates as collateral rates is motivated by market consensus. However, two counterparties might enter a collateral agreement that involves a remuneration of collateral at any other risky rate of their choice. Here we do not assume any requirements on collateral rates. This allows us to cover the quite common situation where the collateral rate agreed between the two counterparties in the CSA is defined by including a real valued spread over some market publicly observed rate, e.g. EONIA -50 bps, where bps stands for basis points.

For the collateral account we have the following equations:

(i) if $C_{t}>0$, then the bank has lent $\psi_{t}^{c}=\psi_{t}^{c, l}<0$ units of the collateral account to the counterparty, i.e.,

$$
\psi_{t}^{c, l} B_{t}^{c, l}=-C_{t}^{+}, t \in[0, T]
$$


(ii) if $C_{t}<0$, then the bank has borrowed $\psi_{t}^{c}=\psi_{t}^{c, b}>0$ units of the collateral account from the counterparty, i.e.,

$$
\psi_{t}^{c, b} B_{t}^{c, b}=C_{t}^{-}, t \in[0, T] .
$$

It is worth noting that we use the following conventions: $x^{+}:=\max \{x, 0\}, x^{-}:=\max \{-x, 0\}$ so that $x=x^{+}-x^{-}$. Note that this is in contrast to the convention adopted e.g. in Burgard and Kjaer 2011a, $2013)$.

2.2.2. Initial margin. The collateralization represented by the variation margin is imperfect, due to the margin period of risk phenomenon: a defaulted counterparty stops posting collateral. However, bankruptcy procedure requires a certain time interval (typically 10 or 20 days) before the close-out payments are performed. This results in a period of time where the value of the transaction oscillates in the absence of an adjustment of the collateral account, hence producing an exposure. This is one of the reasons for the introduction of initial margins, which constitutes a further form of collateral. According to the EMIR regulation, starting from 2020, most agents participating in an OTC transaction will be forced to post initial margin, which constitutes an additional form of collateral. Initial margin, according to Garcia Trillos et al. (2016) is a misnomer, as an initial margin is not only initial, but it is periodically updated during the lifetime of the trade. It is initial in the sense that it is meant to provide a coverage from the initial point in time, where there is a default of the counterparty in a collateralized transaction.

It is important to stress that, differently from variation margin, an initial margin can not be rehypothecated, but it is instead segregated. From the point of view of the wealth dynamics, this means that initial margin received from the counterparty can not be used by the trading desk as a component of the value of the portfolio. However, the received initial margin represents a loan from the counterparty that must be remunerated, hence funding costs related to initial margin will appear in the self-financing condition, see (2.18) and (3.6) for further details.

Remark 2.2. Initial margins are usually quantified by using suitable risk measures, such as value at risk or expected shortfall, as we will specify in (3.24). Expected shortfall is a popular choice to compute the initial margin for credit derivatives, since it is a coherent risk measure. Recently, the International Swaps and Derivatives Association (ISDA) has proposed a novel methodology, the so called Standard Initial Margin Model (SIMM), see ISDA (2018). SIMM provides some standardized formulae to evaluate initial margin on non-cleared derivatives, based on using portfolio sensitivities instead of historical simulations.

We model initial margins with $\mathbb{G}$-adapted processes $I^{T C}=\left(I_{t}^{T C}\right)_{t \in[0, T]}, I^{F C}=\left(I_{t}^{F C}\right)_{t \in[0, T]}$, and we denote by $B^{I, x}, x \in\{l, b\}$, the cash accounts associated to $I^{T C}, I^{F C}$, respectively.

In case the bank is the initial margin provider, the bank posts $I^{T C}$ to the counterparty (TC), i.e.

$$
\psi_{t}^{I, l} B_{t}^{I, l}=-I_{t}^{T C}, t \in[0, T],
$$

or equivalently

$$
-\psi_{t}^{I, l} d B_{t}^{I, l}=r_{t}^{I, l} I_{t}^{T C} d t
$$

In case the bank is the initial margin taker, the bank receives $I^{F C}$ from the counterparty (FC), i.e.

$$
\psi_{t}^{I, b} B_{t}^{I, b}=I_{t}^{F C}, t \in[0, T],
$$


or equivalently

$$
\psi_{t}^{I, b} d B_{t}^{I, b}=r_{t}^{I, b} I_{t}^{F C} d t
$$

We highlight that, contrary to the case of variation margin and in line with current market practice, $I^{T C}$ and $I^{F C}$ are simultaneously active and do not net each other. In the sequel we use $I=\left(I_{t}\right)_{t \in[0, T]}$ as shorthand for both presented or received initial margins.

We now introduce the definition of self-financing strategy in our market model. We recall that we assume the point of view of the bank (i.e. the hedger).

Definition 2.3. A dynamic portfolio, denoted by $\varphi$, is given by

$$
\varphi=\left(\xi^{1}, \ldots, \xi^{d}, \xi^{B}, \xi^{C}, \psi^{1}, \ldots, \psi^{d}, \psi^{B}, \psi^{C}, \psi^{f, b}, \psi^{f, l}, \psi^{c, b}, \psi^{c, l}, \psi^{I, b}, \psi^{I, l}\right)
$$

where

(i) $\xi^{1}, \ldots, \xi^{d}$ are $\mathbb{G}$-predictable processes, denoting the number of shares of the risky primary assets $S^{1}, \ldots, S^{d}$.

(ii) $\xi^{B}, \xi^{C}$ are $\mathbb{G}$-predictable processes, denoting the number of shares of the risky bonds $P^{B}$ and $P^{C}$.

(iii) $\psi^{1}, \ldots, \psi^{d}, \psi^{B}, \psi^{C}$ are $\mathbb{G}$-adapted processes, denoting the number of shares of the repo accounts $B^{1}, \ldots B^{d}, B^{B}, B^{C}$.

(iv) $\psi^{f, b}$ is a $\mathbb{G}$-adapted process, denoting the number of shares of the unsecured funding borrowing cash account $B^{f, b}$.

(v) $\psi^{f, l}$ is a $\mathbb{G}$-adapted process, denoting the number of shares of the unsecured funding lending cash account $B^{f, l}$.

(vi) $\psi^{c, b}$ is a $\mathbb{G}$-adapted process, denoting the number of shares of the collateral borrowing cash account $B^{c, b}$ for the received cash collateral.

(vii) $\psi^{c, l}$ is a $\mathbb{G}$-adapted process, denoting the number of shares of the collateral lending cash account $B^{c, l}$ for the posted cash collateral.

(viii) $\psi^{I, b}$ is a $\mathbb{G}$-adapted process, denoting the number of shares of the initial margin borrowing cash account $B^{I, b}$ for the initial margin received from the counterparty.

(ix) $\psi^{I, l}$ is a $\mathbb{G}$-adapted process, denoting the number of shares of the initial margin lending cash account $B^{I, l}$ for the initial margin posted to the counterparty.

All processes introduced above are such that the stochastic integrals in the sequel are well defined.

Given a dynamic portfolio, we associate it to a financial contract, known in the literature as Credit Support Annex (CSA), see e.g. BCBS (2014).

Definition 2.4. A CSA between the bank and the counterparty is represented by the pair $(C, I)$, where $C$ is the variation margin and $I$ is the initial margin.

Definition 2.5. A collateralized hedger's trading strategy associated to the collateralized contract $\bar{A}$ and the $C S A(C, I)$ is a quintuplet $(x, \varphi, \bar{A}, C, I)$, where $x \in \mathbb{R}$ is the initial endowment, $\varphi$ is a dynamic portfolio as in Definition 2.3 and $I$ is the initial margin.

Definition 2.6. Given the initial endowment $x$, a collateralized hedger's trading strategy $(x, \varphi, \bar{A}, C, I)$ associated to the collateralized contract $\bar{A}$ and the $C S A(C, I)$ is said to be self-financing if, for any $t \in[0, T]$, the wealth process $V_{t}(\varphi)$ given by

$$
V_{t}(\varphi):=\psi_{t}^{f, b} B_{t}^{f, b}+\psi_{t}^{f, l} B_{t}^{f, l}-\left(\psi_{t}^{c, b} B_{t}^{c, b}+\psi_{t}^{c, l} B_{t}^{c, l}+\psi_{t}^{I, l} B_{t}^{I, l}\right)
$$


satisfies

$(2.18)$

$$
\begin{aligned}
V_{t}(\varphi) & =x+\sum_{i=1}^{d} \int_{(0, t]} \xi_{u}^{i}\left(\mu^{i}\left(u, S_{u}\right) d u+\sum_{k=1}^{d} \sigma^{i, k}\left(u, S_{u}\right) d W_{u}^{k, \mathbb{P}}+\kappa^{i}\left(u, S_{u}\right) d u\right) \\
& +\sum_{i=1}^{d} \int_{0}^{t} \psi_{u}^{i} d B_{u}^{i}+\sum_{j \in\{B, C\}} \int_{0}^{t}\left(\xi_{u}^{j} d P_{u}^{j}+\psi_{u}^{j} d B_{u}^{j}\right)-\bar{A}_{t} \\
& +\int_{0}^{t} \psi_{u}^{f, b} d B_{u}^{f, b}+\int_{0}^{t} \psi_{u}^{f, l} d B_{u}^{f, l}-\int_{0}^{t} \psi_{u}^{c, b} d B_{u}^{c, b}-\int_{0}^{t} \psi_{u}^{c, l} d B_{u}^{c, l}-\int_{0}^{t} \psi_{u}^{I, b} d B_{u}^{I, b}-\int_{0}^{t} \psi_{u}^{I, l} d B_{u}^{I, l} .
\end{aligned}
$$

The last two terms in (2.18) represent the cash for the received initial margin. In general, we assume zero initial endowment, $x=0$, i.e., $V_{t}(\varphi)=V_{t}(0, \varphi, \bar{A}, C, I)$ for the sake of simplicity. Note that the sign minus in front of the last term in 2.17) depends on our convention on the collateral. Moreover, we are not including the cash account for the received initial margin. This is due to the fact that the received initial margin is posted in a segregated account and, hence, is not available as a funding asset to the trading desk.

Definition 2.7. A collateralized hedger's trading strategy is admissible if it is self-financing and the associated value process $V(\varphi)$ is bounded from below.

We provide the following definition of arbitrage-free strategy.

Definition 2.8. The market model is arbitrage-free if for any admissible hedger's trading strategy of the form $(0, \varphi, 0,0,0)$ we have either

$$
\mathbb{P}\left[V_{\tau}(0, \varphi, 0,0,0)=0\right]=1 \text { or } \mathbb{P}\left[V_{\tau}(0, \varphi, 0,0,0)<0\right]>0 .
$$

Remark 2.9. In Bielecki and Rutkowski 2015, Definition 3.3) the authors introduce the concept of a market which is said to be arbitrage-free for the hedger with respect to a class of contingent claims. Their definition is formulated in terms of a netted wealth process, which corresponds to a long-short strategy involving the claim $\bar{A}$, where the first position is hedged and the second is unhedged. On the other hand, in Bichuch et al. (2018a) the question concerning absence of arbitrage is first answered in a setting where only the basic traded assets are considered. This is also referred to as absence of arbitrage with respect to the null contract in Bielecki et al. (2018). In our setting, the two approaches coincide.

Assumption 2.10. We assume $r_{t}^{f, l} \leq r_{t}^{f, b}, d \mathbb{P} \otimes d t$-a.s.

We now prove absence of arbitrage for the market model in our setting.

Proposition 2.11. Let Assumption 2.10 hold. Moreover, assume there exists a probability measure $\mathbb{Q} \sim \mathbb{P}$ such that the discounted asset price processes

$$
\tilde{S}_{t}^{i, c l d}:=\frac{S_{t}^{i, c l d}}{B_{t}^{i}}, i=1, \ldots d, \quad \tilde{P}_{t}^{j}:=\frac{P_{t}^{j}}{B_{t}^{j}}, j \in\{B, C\},
$$

are local martingales. Then, the market model is free of arbitrage opportunities.

Proof. See Section A.1 in Appendix A.

From now on, we assume the following. 
Assumption 2.12. There exists an equivalent martingale probability measure $\mathbb{Q} \sim \mathbb{P}$ under which the processes $\tilde{S}_{t}^{i, c l d}, \tilde{P}_{t}^{j}$ in 2.19 are local martingales with dynamics

$$
\begin{aligned}
d \tilde{S}_{t}^{i, c l d} & =\frac{1}{B_{t}^{i}}\left(d S_{t}^{i}-r_{t}^{i} S_{t}^{i} d t+d D_{t}^{i}\right)=\sum_{k=1}^{d} \frac{\sigma^{i, k}\left(t, S_{t}\right)}{B_{t}^{i}} d W_{t}^{k, \mathbb{Q}}, i=1, \ldots, d, \\
d \tilde{P}_{t}^{j} & =\frac{1}{B_{t}^{j}}\left(d P_{t}^{j}-r_{t}^{j} P_{t-}^{j} d t\right)=-\tilde{P}_{t-}^{j} d M_{t}^{j, \mathbb{Q}}, j \in\{B, C\},
\end{aligned}
$$

where

with

$$
M_{t}^{j, \mathbb{Q}}:=H_{t}^{j, \mathbb{Q}}-\int_{0}^{t \wedge \tau^{j}} \lambda_{s}^{j, \mathbb{Q}} d s
$$

$$
\lambda_{t}^{j, \mathbb{Q}}:=r_{t}^{f}-\lambda_{t}^{j, \mathbb{P}}-r_{t}^{j}, t \in[0, T], j \in\{B, C\} .
$$

By Aksamit and Fontana (2019) we obtain that the predictable representation property in $\mathbb{G}$ still holds after the change of measure to $\mathbb{Q}$ with respect to $W^{\mathbb{Q}}, M^{j, \mathbb{Q}}, j \in\{B, C\}$.

Definition 2.13. Let $\beta \geq 0$. The subspace of all $\mathbb{R}^{d}$-valued, $\mathbb{F}$-adapted processes $X$ such that

$$
\mathbb{E}^{\mathbb{Q}}\left[\int_{0}^{T} e^{\beta t}\left|X_{t}\right|^{2} d t\right]<\infty
$$

is denoted by $\mathcal{H}_{\beta, T}^{2, d}(\mathbb{Q})$. We set $\mathcal{H}^{2, d}(\mathbb{Q}):=\mathcal{H}_{0, T}^{2,, d}(\mathbb{Q})$ and

$$
\|X\|_{\mathcal{H}_{\beta, T}^{2, d}}:=\sqrt{\mathbb{E}^{\mathbb{Q}}\left[\int_{0}^{T} e^{\beta t}\left|X_{t}\right|^{2} d t\right] .}
$$

The subspace of all $\mathbb{R}^{d}$-valued, $\mathbb{F}$-adapted processes $X$ such that

$$
\mathbb{E}^{\mathbb{Q}}\left[\sup _{t \in[0, T]} e^{\beta t}\left|X_{t}\right|^{2}\right]<\infty
$$

is denoted by $\mathcal{S}_{\beta, T}^{2, d}(\mathbb{Q})$. We set $\mathcal{S}^{2}(\mathbb{Q}):=\mathcal{S}_{0, T}^{2,1}(\mathbb{Q})$ and

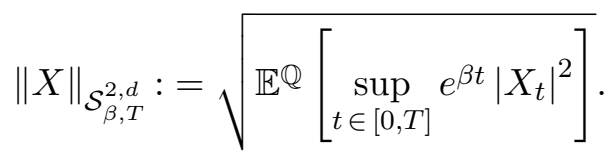

We denote by $\mathcal{H}_{\lambda}^{2,2}(\mathbb{Q})$ the space of $\mathbb{F}$-adapted processes $X$ with values in $\mathbb{R}^{2}$ such that

$$
\mathbb{E}^{\mathbb{Q}}\left[\int_{0}^{T}\left|X_{t}^{1}\right|^{2} \lambda_{t}^{B, \mathbb{Q}}+\left|X_{t}^{2}\right|^{2} \lambda_{t}^{C, \mathbb{Q}} d t\right]<\infty
$$

with

$$
\|X\|_{\mathcal{H}_{\lambda}^{2,2}}=\sqrt{\mathbb{E}^{\mathbb{Q}}\left[\int_{0}^{T}\left|X_{t}^{1}\right|^{2} \lambda_{t}^{B, \mathbb{Q}}+\left|X_{t}^{2}\right|^{2} \lambda_{t}^{C, \mathbb{Q}} d t\right]} .
$$

Assumption 2.14. Assume that the payment stream $A \in \mathcal{S}^{2}(\mathbb{Q})$.

\section{XVA FRAMEWORK}

The main contribution of this Section is manifold. On the one hand, in an arbitrage-free framework we determine the unique solution to the $\mathbb{G}$-BSDE for the valuation of the hedger portfolio $(x, \varphi, \bar{A}, C, I)$, 
consisting of risk-less and risky assets, defaultable bonds and contingent claims. On the other hand, we provide an arbitrage-free framework for clean values and the market practice of using contingent claim specific discounting regimes (CSA discounting) by the introduction of a new value adjustment. Finally, we investigate the shape of the $\mathbb{G}$-BSDE in the realistic situation where the portfolio has a hierarchical structure of sub-portfolios, and consider non-linear effects in portfolio's valuation.

Under Assumption 2.12 the dynamics of a self-financing collateralized trading strategy $(x, \varphi, \bar{A}, C, I)$ is

$$
\begin{aligned}
d V_{t}(\varphi)= & \sum_{i=1}^{d} \xi_{t}^{i} B_{t}^{i} d \tilde{S}_{t}^{i, c l d}+\sum_{j \in\{B, C\}} \xi_{t}^{j} B_{t}^{j} d \tilde{P}_{t}^{j}-d \bar{A}_{t} \\
& +\psi_{t}^{f, l} d B_{t}^{f, l}+\psi_{t}^{f, b} d B_{t}^{f, b}-\psi_{t}^{c, l} d B_{t}^{c, l}-\psi_{t}^{c, b} d B_{t}^{c, b}-\psi_{t}^{I, l} d B_{t}^{I, l}-\psi_{t}^{I, b} d B_{t}^{I, b} .
\end{aligned}
$$

By using the repo constraints (2.9), (2.11), 2.12) and (2.14), the portfolio value satisfies

$$
V_{t}(\varphi)=\psi_{t}^{f, l} B_{t}^{f, l}+\psi_{t}^{f, b} B_{t}^{f, b}+C_{t}+I_{t}^{T C}, t \in[0, T]
$$

since $I^{F C}$ is segregated.

Thanks to 2.10 we obtain the identities

$$
\begin{aligned}
& \psi_{t}^{f, l}=\left(V_{t}(\varphi)-C_{t}-I_{t}^{T C}\right)^{+}\left(B_{t}^{f, l}\right)^{-1}, \\
& \psi_{t}^{f, b}=-\left(V_{t}(\varphi)-C_{t}-I_{t}^{T C}\right)^{-}\left(B_{t}^{f, b}\right)^{-1}
\end{aligned}
$$

for $t \in[0, T]$. Observe that by (2.11) and (2.12)

$$
\begin{aligned}
& -\psi_{t}^{c, l} d B_{t}^{c, l}=-\psi_{t}^{c, l} r_{t}^{c, l} B_{t}^{c, l} d t=+r_{t}^{c, l} C_{t}^{+} d t, \\
& -\psi_{t}^{c, b} d B_{t}^{c, b}=-\psi_{t}^{c, b} r_{t}^{c, b} B_{t}^{c, b} d t=-r_{t}^{c, b} C_{t}^{-} d t,
\end{aligned}
$$

respectively. By (3.2), (3.3), 3.4, (3.5), 2.14) and (2.16), we can rewrite the wealth dynamics as follows

$$
\begin{aligned}
d V_{t}(\varphi) & =\sum_{i=1}^{d} \xi_{t}^{i} B_{t}^{i} d \tilde{S}_{t}^{i, c l d}+\sum_{j \in\{B, C\}} \xi_{t}^{j} B_{t}^{j} d \tilde{P}_{t}^{j}-d \bar{A}_{t} \\
& +\left[r_{t}^{f, l}\left(V_{t}(\varphi)-C_{t}-I_{t}^{T C}\right)^{+}-r_{t}^{f, b}\left(V_{t}(\varphi)-C_{t}-I_{t}^{T C}\right)^{-}\right. \\
& \left.+r_{t}^{c, l} C_{t}^{+}-r_{t}^{c, b} C_{t}^{-}+r_{t}^{I, l} I_{t}^{T C}-r_{t}^{I, b} I_{t}^{F C}\right] d t
\end{aligned}
$$

We now introduce for convenience an auxiliary artificial interest rate process $r=\left(r_{t}\right)_{t \in[0, T]}$, assumed to be right-continuous, bounded and $\mathbb{F}$-adapted. This rate is not necessarily linked to a traded asset, but it can be interpreted as an interest rate level, used to express all other rates as spreads over this artificial rate. When needed, we will explicitly state when the rate $r$ becomes a market rate. Using the artificial rate $r$, we can conveniently rewrite the portfolio dynamics as follows

$$
\begin{aligned}
d V_{t}(\varphi) & =\sum_{i=1}^{d} \xi_{t}^{i} B_{t}^{i} d \tilde{S}_{t}^{i, c l d}+\sum_{j \in\{B, C\}} \xi_{t}^{j} B_{t}^{j} d \tilde{P}_{t}^{j}-d \bar{A}_{t} \\
& +\left[\left(r_{t}^{f, l}-r_{t}\right)\left(V_{t}(\varphi)-C_{t}-I_{t}^{T C}\right)^{+}-\left(r_{t}^{f, b}-r_{t}\right)\left(V_{t}(\varphi)-C_{t}-I_{t}^{T C}\right)^{-}\right. \\
& \left.+\left(r_{t}^{c, l}-r_{t}\right) C_{t}^{+}-\left(r_{t}^{c, b}-r_{t}\right) C_{t}^{-}+\left(r_{t}^{I, l}-r_{t}\right) I_{t}^{T C}-r_{t}^{I, b} I_{t}^{F C}+r_{t} V_{t}(\varphi)\right] d t
\end{aligned}
$$




$$
\begin{aligned}
& =\sum_{i, k=1}^{d} \xi_{t}^{i} \sigma^{i, k}\left(t, S_{t}\right) d W_{t}^{k, \mathbb{Q}}-\sum_{j \in\{B, C\}} \xi_{t}^{j} B_{t}^{j} \tilde{P}_{t-}^{j} d M_{t}^{j, \mathbb{Q}}-d \bar{A}_{t} \\
& +\left[\left(r_{t}^{f, l}-r_{t}\right)\left(V_{t}(\varphi)-C_{t}-I_{t}^{T C}\right)^{+}-\left(r_{t}^{f, b}-r_{t}\right)\left(V_{t}(\varphi)-C_{t}-I_{t}^{T C}\right)^{-}\right. \\
& \left.+\left(r_{t}^{c, l}-r_{t}\right) C_{t}^{+}-\left(r_{t}^{c, b}-r_{t}\right) C_{t}^{-}+\left(r_{t}^{I, l}-r_{t}\right) I_{t}^{T C}-r_{t}^{I, b} I_{t}^{F C}+r_{t} V_{t}(\varphi)\right] d t,
\end{aligned}
$$

where we added and subtracted the term $r_{t} V_{t}(\varphi) d t$ and used Assumption 2.12.

Remark 3.1. The term $\left(r_{t}^{I, l}-r_{t}\right) I_{t}^{T C}$ measures a funding benefit from the posted initial margin over the reference rate level $r$. We would like to stress that, in general, spreads over $r$ can be negative, representing that we may have funding costs, even when the bank is collateral provider. Such a situation is faced by banks, which clear swaps with the London Clearing House (LCH). If $r$ is chosen to represent the EONIA overnight rate, then the rate applied by $\mathrm{LCH}$ is $r^{I, l}=r-58 \mathrm{bps}$, where bps stands for basis point:1. On top of such a negative benefit, the bank needs to take into account the cost of raising the amount $I^{T C}$, hence initial margin can generate funding costs in both directions, from the point of view of fund-raising and from the point of view of collateral remuneration, hence representing a significant source of costs for the bank.

In case of default, cashflows are exchanged between the surviving agent and the liquidators of the defaulted agent. Here we use the term agent as a placeholder for the bank or for the counterparty. Due to the exchange of cashflows at default time, agents need to perform a valuation of the position at a random time represented by the close-out condition, see Bichuch et al. (2018a) Section 3.4. The object of the analysis can be the value in the absence of counterparty risk (referred to in the literature as risk-free close-out) or the value of the trade including the price adjustments due to counterparty risk and funding (risky close-out), see e.g. Brigo and Morini (2018). A risky close-out condition guarantees that the surviving counterparty can ideally fully substitute the transaction with a new trade entered with another counterparty with the same credit quality. This comes at the price of a significant increase of the complexity of the valuation equations. Current market practice and the existing literature mainly focus on the case of risk-free close-out value.

Definition 3.2. Let $0<R^{j}<1, j \in\{B, C\}$, be the recovery rates of the bank and the counterparty, respectively. The close-out condition $\theta_{\tau}\left(\mathcal{V}, C, I^{T C}, I^{F C}\right)$, expressed from the bank's perspective, is defined by

$$
\begin{aligned}
\theta_{\tau}\left(\mathcal{V}, C, I^{T C}, I^{F C}\right):= & \mathcal{V}_{\tau}+1_{\left\{\tau^{C}<\tau^{B}\right\}}\left(1-R^{C}\right)\left(\mathcal{V}_{\tau}-C_{\tau-}+I_{\tau-}^{F C}\right)^{-} \\
& -1_{\left\{\tau^{B}<\tau^{C}\right\}}\left(1-R^{B}\right)\left(\mathcal{V}_{\tau}-C_{\tau-}-I_{\tau-}^{T C}\right)^{+}
\end{aligned}
$$

We restate the portfolio dynamics under $\mathbb{Q}$ in the form of a BSDE. We set

$$
\begin{aligned}
Z_{t}^{k} & :=\frac{\sum_{i=1}^{d} \xi_{t}^{i} \sigma^{i, k}\left(t, S_{t}\right)}{B_{t}^{i}} \\
U_{t}^{j} & :=-\xi_{t}^{j} \tilde{P}_{t-}^{j} \\
f\left(t, V, C, I^{T C}, I^{F C}\right) & :=-\left[\left(r_{t}^{f, l}-r_{t}\right)\left(V_{t}(\varphi)-C_{t}-I_{t}^{T C}\right)^{+}-\left(r_{t}^{f, b}-r_{t}\right)\left(V_{t}(\varphi)-C_{t}-I_{t}^{T C}\right)^{-}\right. \\
& \left.+\left(r_{t}^{c, l}-r_{t}\right) C_{t}^{+}-\left(r_{t}^{c, b}-r_{t}\right) C_{t}^{-}+\left(r_{t}^{I, l}-r_{t}\right) I_{t}^{T C}-r_{t}^{I, b} I_{t}^{F C}\right] .
\end{aligned}
$$

$\overline{1_{\text {see https://www.lch.com/risk-collateral-management/ltd-collateral-management/ltd-fees-collateral }}}$ 
Under the close-out condition $(3.8)$, the $\mathbb{G}$-BSDE for the portfolio's dynamics in 3.7$)$ under $\mathbb{Q}$ has then the form

$$
\left\{\begin{array}{l}
-d V_{t}(\varphi)=d \bar{A}_{t}+\left(f\left(t, V, C, I^{T C}, I^{F C}\right)-r_{t} V_{t}(\varphi)\right) d t-\sum_{k=1}^{d} Z_{t}^{k} d W_{t}^{k, \mathbb{Q}}-\sum_{j \in\{B, C\}} U_{t}^{j} d M_{t}^{j, \mathbb{Q}} \\
V_{\tau}(\varphi)=\theta_{\tau}\left(\mathcal{V}, C, I^{T C}, I^{F C}\right),
\end{array}\right.
$$

for $t \leq \tau$. We prove in Theorem 3.16 that there exists a unique solution $(V, Z, U)$ for the $\mathbb{G}$-BSDE (3.10), and the process $V$ assumes the following form on $\{\tau>t\}$

$$
V_{t}(\varphi)=B_{t}^{r} \mathbb{E}^{\mathbb{Q}}\left[\int_{(t, \tau \wedge T]} \frac{d \bar{A}_{u}}{B_{u}^{r}}+\int_{t}^{\tau \wedge T} \frac{f\left(u, V, C, I^{T C}, I^{F C}\right)}{B_{u}^{r}} d u+1_{\{\tau \leq T\}} \frac{\theta_{\tau}\left(\mathcal{V}, C, I^{T C}, I^{F C}\right)}{B_{\tau}^{r}} \mid \mathcal{G}_{t}\right],
$$

where $B_{t}^{r}:=\exp \left(\int_{0}^{t} r_{u} d u\right), t \in[0, T]$.

Remark 3.3. The $\mathbb{G}$-BSDE 3.10 is in line with the current market practice on xVA. Regulatory changes in the bankruptcy procedures can be encoded via changes in the terminal condition (i.e. the close-out condition) whereas new funding policies can be captured by suitable adjustments of the driver.

In order to solve (3.10) we first have to specify our choice for $\mathcal{V}$ in the close-out condition (3.8). To this purpose, we need the results of the following Section.

3.1. Clean Value under $\mathbb{F}$. A financial product can be traded between any two counterparties. Since every agent has a different credit quality and different funding costs, this means in general that a single product (e.g. a 10 year EUR swap) has as many potential values as the number of possible combinations of agents in the market. It would be highly impractical for a broker to publish all possible market quotes for all possible counterparties. Publicly observable market quotes provided by data providers do not take into account xVA frictions and are typically given by a single value (more precisely a bid and offer price) called clean price. We now provide an arbitrage-free valuation for clean prices consistent with the current market practice for market quotes ${ }^{2}$.

A clean price is an ideal value process that would be acceptable between two agents entering a perfectly collateralized transaction. Perfect collateralization however is not enough to produce a clean price: we also need to explicitly assume that the two agents entering the transaction are default-free. This is necessary because, even in the presence of a perfect ideal collateral agreement, counterparty risk is not perfectly annihilated: when a counterparty defaults, she stops posting collateral.

However, default is not automatically legally recognized: typically, bankruptcy procedures require some days (e.g. 10 or 20 days) before the close-out payments are exchanged. This creates a period of time where the counterparty is not officially defaulted but without any collateral adjustment. Such period of time is known as margin period of risk. During such interval of time the value of the claim deviates from the value of the collateral account thus creating a credit exposure.

Hence, to preclude margin period of risk and obtain the ideal clean price process, we consider a parallel market model with perfect collateralization but no default risk.

\footnotetext{
${ }^{2}$ Some authors criticize the idea of a price decomposition in terms of clean value and an adjustment process, see e.g. Bielecki et al. (2018). A situation where the decomposition is not justified is given e.g. by a contingent claim whose dividend process depends in a non-linear way on the strategy of the hedger in a fully non-linear market, where we have bid offer spread in all rates, including the repo rates of the assets (which are zero in our case). However, payoffs traded on the market do not usually feature such non-linear effects. Furthermore, publicly available market quotes are not influenced by counterparty credit risk.
} 
Assumption 3.4 (Clean market). A clean market under $\mathbb{F}$ without bid-offer spreads is defined by

(i) no bid-offer spread in the funding accounts, i.e., $r_{t}^{f, l}=r_{t}^{f, b}=r^{f}$;

(ii) no bid-offer spread in the collateral accounts, i.e., $r_{t}^{c, l}=r_{t}^{c, b}=r^{c}$;

(iii) the collateral rate is equal to the fictious rate, i.e., $r^{c}=r$;

(iv) there is no default, i.e. $\tau^{B}=\tau^{C}=\infty$ and risky bonds are excluded from the market;

(v) there is no exchange of initial margin;

(vi) perfect collateralization, i.e., $\hat{V}_{t} \equiv C_{t}$, for all $t \in[0, T]$, where we use $\hat{V}$ to denote the value process of a collateralized hedging strategy in the fictious market without default-risk.

Note that (vi) in Assumption 3.4 implies that the portfolio weights in the cash accounts are of the form

$$
\psi_{t}^{c}=-\frac{\hat{V}_{t}}{B_{t}^{c}}, \quad \psi_{t}^{f} \equiv 0, \text { for all } t \in[0, T]
$$

meaning that the position is totally funded by the collateralization scheme, and $\hat{V}=\left(\hat{V}_{t}\right)_{[0, T]}$ is an $\mathbb{F}$-adapted process.

The dynamics of the clean portfolio value $\hat{V}$ resulting from (3.7) and Assumption 3.4 are given by

$$
d \hat{V}_{t}(\varphi)=\sum_{k=1}^{d} \hat{Z}_{t}^{k} d W_{t}^{k, \mathbb{Q}}-d A_{t}+r_{t} \hat{V}_{t}(\varphi) d t, \text { where } \hat{Z}_{t}^{k}:=\sum_{i=1}^{d} \hat{\xi}_{t}^{i} \sigma^{i, k}\left(t, S_{t}\right) .
$$

Inserting the terminal condition $\hat{V}_{T}=0$, we can rewrite the $\mathbb{F}$-dynamics $(3.12)$ for $\hat{V}$ under $\mathbb{Q}$ in the classical $\mathbb{F}$-BSDE form

$$
\left\{\begin{array}{l}
-d \hat{V}_{t}(\varphi)=d A_{t}-r_{t} \hat{V}_{t}(\varphi) d t-\sum_{k=1}^{d} \hat{Z}_{t}^{k} d W_{t}^{k, \mathbb{Q}} \\
\hat{V}_{T}(\varphi)=0
\end{array}\right.
$$

We now prove existence and uniqueness for the solution of 3.13 .

Theorem 3.5. Under Assumption 2.14 on A, there exists a unique solution $(\hat{V}, \hat{Z}) \in \mathcal{S}^{2}(\mathbb{Q}) \times$ $\mathcal{H}^{2, d}(\mathbb{Q})$ to the clean $\mathbb{F}-B S D E(3.13$.

Proof. We note that the clean BSDE (3.13) is similar to the linear BSDE studied e.g. in El Karoui et al. (1997), where the driver is the multidimensional Brownian motion $\left(W^{1, \mathbb{Q}}, \ldots, W^{d, \mathbb{Q}}\right)^{\top}$.

We can apply Nie and Rutkowski (2016. Theorem 4.1) by observing that $M=W^{\mathbb{Q}}, Q_{t}=t, U=A$, $\hat{V}=Y$ and $h\left(t, Y_{t}, Z_{t}\right)=-r_{t} \hat{V}_{t}$, which clearly fulfills the uniform Lipschitz condition. Also the condition $h(\cdot, 0,0) \in \mathcal{S}^{2}(\mathbb{Q})$ is trivially satisfied. We also observe that $\mathbb{X}=S=\operatorname{diag}\left(S^{1}, \ldots, S^{d}\right)$, hence we have $m_{t}=\sigma\left(t, S_{t}\right)$, so that $\gamma_{t}=S_{1}^{-1} \sigma\left(t, S_{t}\right)$, for $\gamma$ satisfying the ellipticity condition A.2. According to Theorem A.7 we have $\hat{V} \in \mathcal{H}^{2}(\mathbb{Q})$ and $\hat{V}-A \in \mathcal{S}^{2}(\mathbb{Q})$. Now, Assumption 2.14 allows us to conclude that also $\hat{V} \in \mathcal{S}^{2}(\mathbb{Q})$.

Next we show that the process $\hat{V}$ in Theorem 3.5 provides the arbitrage-free price for the contract with cashflow stream $A$. 
Theorem 3.6. Let $\mathbb{Q} \sim \mathbb{P}$ be an equivalent probability measure such that all processes $\tilde{S}^{i, c l d}, i=$ $1, \ldots, d$, are local $\mathbb{Q}$-martingales. Let $(\hat{V}, \hat{Z})$ be the unique solution of (3.13). Then, under Assumption 2.14 on $A$, we have

$$
\hat{V}_{t}(\varphi):=\mathbb{E}^{\mathbb{Q}}\left[B_{t}^{r} \int_{(t, T]} \frac{d A_{u}}{B_{u}^{r}} \mid \mathcal{F}_{t}\right], \text { for all } t \in[0, T] .
$$

Proof. The proof follows because $\hat{Z} \in \mathcal{H}^{2, d}(\mathbb{Q})$ by Theorem 3.5, $B$ is bounded and thanks to Assumption 2.14 .

From now on, we assume to work with the càdlàg version of $\hat{V}$.

Remark 3.7. Here we introduce the concept of clean value by means of a replicating strategy in a fictious idealized market. Our constructive approach is in line with the current market standard and the concept of third-party valuation of Bichuch et al. (2018a). Formula (3.14) encodes the idea of CSA discounting. Since the rate $r$ is the remuneration of collateral in a stylized perfect collateral agreement, we do not need to postulate the existence of a risk-free rate. Bichuch et al. (2018a) define the clean value by introducing an additional valuation measure different from $\mathbb{Q}$. Working with the pricing measure $\mathbb{Q}$ also avoids the issue of estimating parameters under different measures.

So far, our discussion of the clean market focused on a dividend process specified under the reference filtration $\mathbb{F}$. As stressed e.g. in Crépey (2015b), this assumption is too restrictive to e.g. cover credit derivatives or wrong-way risk. Though, our objective is to focus on multiple aggregation levels and different discounting regimes, hence we choose to avoid the technicalities that are involved in generalizations of the immersion hypothesis.

3.2. Portfolio valuation via $\mathbb{G}-B S D E$. We now discuss existence and uniqueness of the solution for the $\mathbb{G}$-BSDE (3.10) by following the approach of Crépey (2015b). To this purpose we use the results on the clean value of Section 3.1 and the following Assumption.

Assumption 3.8. We assume a risk-free close-out valuation under $\mathbb{F}$, namely we set $\mathcal{V}_{t}=\hat{V}_{t}(\varphi)$ in the close-out condition (3.8).

Definition 3.9. We define the following valuation adjustments:

$$
\begin{aligned}
C V A_{t} & :=B_{t}^{r} \mathbb{E}^{\mathbb{Q}}\left[1_{\left\{\tau^{C}<\tau^{B}\right\}}\left(1-R^{C}\right) \frac{1}{B_{\tau}^{r}}\left(\hat{V}_{\tau}(\varphi)-C_{\tau-}+I_{\tau-}^{F C}\right)^{-} \mid \mathcal{G}_{t}\right], \\
D V A_{t} & :=B_{t}^{r} \mathbb{E}^{\mathbb{Q}}\left[1_{\left\{\tau^{B}<\tau^{C}\right\}}\left(1-R^{B}\right) \frac{1}{B_{\tau}^{r}}\left(\hat{V}_{\tau}(\varphi)-C_{\tau-}-I_{\tau-}^{T C}\right)^{+} \mid \mathcal{G}_{t}\right], \\
F V A_{t} & :=B_{t}^{r} \mathbb{E}^{\mathbb{Q}}\left[\int_{t}^{\tau} \frac{\left(r_{u}^{f, l}-r_{u}\right)\left(V_{u}(\varphi)-C_{u}-I_{u}^{T C}\right)^{+}-\left(r_{u}^{f, b}-r_{u}\right)\left(V_{u}(\varphi)-C_{u}-I_{u}^{T C}\right)^{-}}{B_{u}^{r}} d u \mid \mathcal{G}_{t}\right], \\
\operatorname{ColV} A_{t} & :=B_{t}^{r} \mathbb{E}^{\mathbb{Q}}\left[\int_{t}^{\tau} \frac{\left(r_{u}^{c, l}-r_{u}\right) C_{u}^{+}-\left(r_{u}^{c, b}-r_{u}\right) C_{u}^{-}}{B_{u}^{r}} d u \mid \mathcal{G}_{t}\right], \\
M V A_{t} & :=B_{t}^{r} \mathbb{E}^{\mathbb{Q}}\left[\int_{t}^{\tau} \frac{\left(r_{u}^{I, l}-r_{u}\right) I_{u}^{T C}-r_{u}^{I, b} I_{u}^{F C}}{B_{u}^{r}} d u \mid \mathcal{G}_{t}\right] .
\end{aligned}
$$

On $\{\tau>t\}$, we define

$$
X V A_{t}:=-C V A_{t}+D V A_{t}+F V A_{t}+\operatorname{Col} V A_{t}+M V A_{t},
$$


and set

$$
X V A_{\tau}=-\theta_{\tau}+\hat{V}_{\tau} \text { on }\{\tau \leq t\}
$$

where $\theta_{\tau}$ is defined in (3.8).

Note that the FVA term in Definition 3.9 has a recursive nature, see also Piterbarg $(2010)$. The exposure is proportional to the full value of the transaction $V$ and not only to the clean value $\hat{V}$. This implies a high complexity of the numerical scheme. Some practitioner's papers, such as Burgard and Kjaer (2013), avoid the recursivity issue by means of ad-hoc choices of the funding strategies, such as the funding strategy called semi-replication with no shortfall on default. However, the bank usually needs to fund the clean value and the value adjustments. Hence, this feature cannot be ignored in a comprehensive mathematical model.

Example 3.10. Set $I_{t}^{T C}=I_{t}^{F C}=0, r^{f, b}=r^{f, l}=r^{f}, r^{c, b}=r^{c, l}=r^{c}$ and $\tau^{C}=\tau^{B}=\infty$. Then the driver of the full BSDE is given by

$$
f(t, V, C, 0):=-\left(\left(r_{t}^{f}-r_{t}\right)\left(V_{t}(\varphi)-C_{t}\right)+\left(r_{t}^{c}-r_{t}\right) C_{t}\right), t \in[0, T] .
$$

In this case, the integral representation (3.11) of $V$ is of the form

$$
V_{t}(\varphi)=B_{t}^{r} \mathbb{E}^{\mathbb{Q}}\left[\int_{(t, T]} \frac{d A_{u}}{B_{u}^{r}}+\int_{t}^{T} \frac{f(u, V, C, 0)}{B_{u}^{r}} d u \mid \mathcal{F}_{t}\right], t \in[0, T] .
$$

If we set $r_{t}=r_{t}^{f} d \mathbb{P} \otimes d t$-a.s. then we obtain by (3.17) that

$$
V_{t}(\varphi)=B_{t}^{r^{f}} \mathbb{E}^{\mathbb{Q}}\left[\int_{(t, T]} \frac{d A_{u}}{B_{u}^{r^{f}}}+\int_{t}^{T}\left(r_{u}^{f}-r_{u}^{c}\right) \frac{C_{u}}{B_{u}^{r^{f}}} d u \mid \mathcal{F}_{t}\right], t \in[0, T] .
$$

This corresponds to equation (3) in Piterbarg (2010). If we set $r_{t}=r_{t}^{c} d \mathbb{P} \otimes d t$-a.s. in (3.17), we obtain

$$
V_{t}(\varphi)=B_{t}^{r^{c}} \mathbb{E}^{\mathbb{Q}}\left[\int_{(t, T]} \frac{d A_{u}}{B_{u}^{r^{c}}}-\int_{t}^{T}\left(r_{u}^{f}-r_{u}^{c}\right) \frac{\left(V_{t}(\varphi)-C_{u}\right)}{B_{u}^{r^{c}}} d u \mid \mathcal{F}_{t}\right], t \in[0, T],
$$

which corresponds to equation (5) in Piterbarg (2010).

From Assumption 3.8 we have $\mathcal{V}=\hat{V}$. Since $\hat{V}$ is an $\mathbb{F}$-adapted càdlàg process, we know from Crépey 2015b, Lemma 2.1) that $\Delta \hat{V}_{\tau}=0$ under the Hypothesis 2.1 between $\mathbb{F}$ and $\mathbb{G}$. The same argument holds true for the collateral process $C$, which we assumed to be a Lipschitz function of the clean value, and for the initial margin $I$, be it posted or received. From now on we identify the clean value $\hat{V}$, the collateral process $C$ and the initial margin $I$ with their left limits. We set

$$
\begin{aligned}
\theta_{t}^{C} & :=\left(1-R^{C}\right)\left(\hat{V}_{t}-C_{t}+I_{t}^{F C}\right)^{-}, \\
\theta_{t}^{B} & :=\left(1-R^{B}\right)\left(\hat{V}_{t}-C_{t}-I_{t}^{T C}\right)^{+},
\end{aligned}
$$

Definition 3.11. We call pre-default xVA-BSDE the following $\mathbb{F}$-BSDE on $[0, T]$ with null terminal condition in $T$ :

$$
\left\{\begin{array}{l}
-d \overline{X V A}_{t}=\bar{f}\left(\hat{V}_{t}-\overline{X V A}_{t}\right) d t-\sum_{k=1}^{d} \bar{Z}_{t}^{k} d W_{t}^{k, \mathbb{Q}} \\
\overline{X V A}_{T}=0
\end{array}\right.
$$

where

$$
\bar{f}\left(\hat{V}_{t}-\overline{X V A}_{t}\right):=-f\left(t, \hat{V}-\overline{X V A}, C, I^{T C}, I^{F C}\right)-\left(r_{t}+\lambda_{t}^{C, \mathbb{Q}}+\lambda_{t}^{B, \mathbb{Q}}\right) \overline{X V A_{t}}-\lambda_{t}^{C, \mathbb{Q}} \theta_{t}^{C}+\lambda_{t}^{B, \mathbb{Q}} \theta_{t}^{B},
$$


for $\theta^{B}, \theta^{C}$ defined as in (3.21) and $\lambda_{t}^{j, \mathbb{Q}}=r_{t}^{f}-\lambda_{u}^{j, \mathbb{P}}-r_{t}^{j}, t \in[0, T], j \in\{B, C\}$.

We now discuss existence and uniqueness for the solution to 3.22 . In the following, we use subscripts of the form $t: T$ to indicate the dependence on the path of a process from $t$ up to $T$. First, we observe that the driver (3.23) also depends on the initial margins. We set

$$
I_{s}^{i}:=\rho_{s}\left(\hat{V}_{s: T}-\overline{X V A}_{s: T}\right)_{s \in[t, T]}, i \in\{T C, F C\},
$$

where $\overline{X V A}_{t: T}:=\left(\overline{X V A}_{s}\right)_{s \in[t, T]}$ is the process defining the pre-default value adjustment and $\hat{V}_{t: T}:=$ $\left(\hat{V}_{s}\right)_{s \in[t, T]}$ is the clean value assumed to be a given exogenous process in $\mathcal{S}^{2}(\mathbb{Q})$, both evaluated up to the contract's maturity, since they are used to measure the potential future exposure. For each $t \in[0 . T], \rho_{t}=\rho(\omega, t ; x)$, is a risk measure. For the sake of simplicity, we assume the same $\rho$ for both $i \in\{T C, F C\}$. This hypothesis can be easily generalized.

We also assume the following

Assumption 3.12. (i) For any $X, Y \in \mathcal{S}^{2}(\mathbb{Q})$, the process $\left(\rho_{t}\left(X_{t: T}-Y_{t: T}\right)\right)_{t \in[0, T]}$ is in $\mathcal{H}^{2,1}(\mathbb{Q})$. There exists a constant $C_{\rho}>0$ and a family of measures $\left(\nu_{s}\right)_{s \in[0, T]}$ on $\mathbb{R}$ such that $\nu_{t}([t ; T])=$ 1 , for every $t \in[0, T]$, and, for any $x, y^{1}, y^{2} \in \mathcal{S}^{2}(\mathbb{Q})$, we have

$$
\left|\rho_{t}\left(x_{t: T}-y_{t: T}^{1}\right)-\rho_{t}\left(x_{t: Y}-y_{t: T}^{2}\right)\right| \leq C_{\rho} \mathbb{E}\left[\int_{t}^{T}\left|y_{s}^{1}-y_{s}^{2}\right| \nu_{t}(d s) \mid \mathcal{F}_{t}\right], d t \otimes d \mathbb{P} \text { a.e. }
$$

(ii) we assume that $f$ satisfies Assumption A.8 with $y=\hat{V}-\overline{X V A}^{i}, z=C$ and $\lambda=\rho^{i}$, for $i=1,2$.

Lemma 3.13. Let $\left(\overline{X V A}^{i}, \bar{Z}^{i}\right) \in \mathcal{S}^{2}(\mathbb{Q}) \times \mathcal{H}^{2, d}(\mathbb{Q}), i=1,2$, be solutions to the $\mathbb{F}$-BSDE (3.22), with $f^{i}\left(t, \hat{V}-\overline{X V A}^{i}, C, \rho^{i}\right)$ satisfying Assumption 3.12, for $i=1,2$.

Moreover, define

$$
\begin{aligned}
& \delta \overline{X V A}:=\overline{X V A}^{1}-\overline{X V A}^{2}, \\
& \delta \bar{Z}:=\bar{Z}^{1}-\bar{Z}^{2}, \\
& \delta f:=f^{1}\left(\hat{V}-\overline{X V A}^{2}\right)-f^{2}\left(\hat{V}-\overline{X V A}^{2}\right), \\
& \delta \rho:=\rho_{s}^{1}\left(\hat{V}_{s: T}-\overline{X V A}_{s: T}^{2}\right)-\rho_{s}^{2}\left(\hat{V}_{s: T}-\overline{X V A}_{s: T}^{2}\right),
\end{aligned}
$$

for any $s \in[t, T]$. Then, there exists a constant $c>0$ such that for $\mu>0$, we have for $\beta$ large enough

$$
\begin{aligned}
\|\delta \overline{X V A}\|_{\mathcal{S}_{\beta, T}^{2}}^{2} & \leq c\left[e^{\beta T} \mathbb{E}\left[|\delta \xi|^{2}\right]+\frac{1}{\mu^{2}}\left(\left\|\delta_{2} f\right\|_{\mathcal{H}_{\beta, T}^{2, d}}^{2}+C_{f^{1}}\left\|\delta_{2} \rho\right\|_{\mathcal{H}_{\beta, T}^{2, d}}^{2}\right)\right], \\
\|\delta \bar{Z}\|_{\mathcal{H}_{\beta, T}^{2, d}}^{2} & \leq c\left[e^{\beta T} \mathbb{E}\left[|\delta \xi|^{2}\right]+\frac{1}{\mu^{2}}\left(\left\|\delta_{2} f\right\|_{\mathcal{H}_{\beta, T}^{2, d}}^{2}+C_{f^{1}}\|\delta \rho\|_{\mathcal{H}_{\beta, T}^{2, d}}^{2}\right)\right],
\end{aligned}
$$

where $\|\delta \overline{X V A}\|_{\mathcal{S}_{\beta, T}^{2}}$ and $\|\delta \bar{Z}\|_{\mathcal{H}_{\beta, T}^{2, d}}$ are defined in 2.23) and 2.21), respectively.

Proof. First, we observe that $f^{i}\left(\hat{V}-\overline{X V A}^{i}\right), i=1,2$, given in $(3.23)$, consists of three terms. The first one is the full $\mathbb{G}$-BSDE driver $f$, given in $(3.9 \mathrm{c})$ and expressed in terms of the collateral $C$, which is a Lipschitz function of the clean value by definition, and the (posted/received) initial margin $I$, which is Lipschitz by (3.24) and (3.25). The second term depends on the short rate $r$ and the jump intensities $\lambda^{B, \mathbb{Q}}, \lambda^{C, \mathbb{Q}}$, which are bounded by definition. The last term relies upon the close-out conditions $\theta^{B}, \theta^{C}$ given in (3.21), which are Lipschitz functions, by following the same arguments as before. Therefore, the driver satisfies Assumption A.8. Moreover, we observe that, for any $\left(\overline{X V A}^{1}, \bar{Z}^{1}\right),\left(\overline{X V A}^{1}, \bar{Z}^{1}\right) \in$ 
$\mathcal{S}^{2}(\mathbb{Q}) \times \mathcal{H}^{2, d}(\mathbb{Q})$, we have

$$
\begin{aligned}
\mid f^{1}(\hat{V} & \left.-\overline{X V A}^{1}\right)-f^{2}\left(\hat{V}-\overline{X V A}^{2}\right) \mid \\
& \leq\left|f^{1}\left(\hat{V}-\overline{X V A}^{1}\right)-f^{1}\left(\hat{V}-\overline{X V A}^{2}\right)\right|+\left|f^{1}\left(\hat{V}-\overline{X V A}^{2}\right)-f^{2}\left(\hat{V}-\overline{X V A}^{2}\right)\right| \\
& \leq\left|f^{1}\left(s, \hat{V}-\overline{X V A}^{1}, C, \rho^{1}\left(\hat{V}_{s: T}-\overline{X V A}_{s: T}^{1}\right)\right)-f^{1}\left(s, \hat{V}-\overline{X V A}^{2}, C, \rho^{1}\left(\hat{V}_{s: T}-\overline{X V A}_{s: T}^{2}\right)\right)\right| \\
& +\left|\left(r_{s}+\lambda_{s}^{C, \mathbb{Q}}+\lambda_{s}^{B, \mathbb{Q}}\right)\left(\overline{X V A}^{1}-\overline{X V A}^{2}\right)\right|+|\delta f| \\
& \leq\left(\bar{C}_{f^{1}}+\bar{C}_{s}\right)(|\delta \overline{X V A}|)+\bar{C}_{f^{1}}\left(\left|\rho^{1}\left(\hat{V}_{s: T}-\overline{X V A}_{s: T}^{1}\right)-\rho^{1}\left(\hat{V}_{s: T}-\overline{X V A}_{s: T}^{2}\right)\right|+|\delta \rho|\right)+|\delta f|
\end{aligned}
$$

where the last inequality holds true thanks to Assumption A.8, adding and subtracting $\rho^{1}\left(\hat{V}_{s: T}-\right.$ $\overline{X V A}_{s: T}^{2}$ ) and choosing a suitable constant $\bar{C}_{s} \geq r_{s}+\lambda_{s}^{C, \mathbb{Q}}+\lambda_{s}^{B, \mathbb{Q}}$.

The result follows by applying the argument provided in the proof of Crépey et al. (2020, Lemma 3.1) with terminal condition $\xi=0$.

Proposition 3.14. Under Assumptions 2.14, A.8 and 3.12, the $\mathbb{F}-B S D E(3.22)$ is well posed and has a unique solution $(\overline{X V A}, \bar{Z}) \in \mathcal{S}^{2}(\mathbb{Q}) \times \mathcal{H}^{2, d}(\mathbb{Q})$.

Proof. The proof is analogous to the one in Crépey et al. (2020, Theorem 3.1) by using the estimates (3.26) and (3.27).

Now, given the uniqueness of the solution to 3.22 we have the following result.

Proposition 3.15. Let $(\overline{X V A}, \bar{Z})$ be the unique solution of the pre-default XVA-BSDE $(3.22)$. Define

$$
X_{t}:=\overline{X V A}_{t} J_{t}+1_{\{\tau \leq t\}} \vartheta_{\tau}, t \in[0, \tau \wedge T],
$$

where $J_{t}:=1_{\{t<\tau\}}=1-H_{t}$ and $\vartheta_{t}:=-\theta_{t}+\hat{V}_{t}, t \in[0, T]$. Then, under Under Assumptions 2.14 and 3.12, the process $(X, \bar{Z}, \tilde{U})$ solves the $\mathbb{G}-B S D E$ on $\{\tau>t\}$

$$
\begin{cases}-d X_{t} & =-\left[f\left(t, \hat{V}-X V A, C, I^{T C}, I^{F C}\right)+r_{t} X V A_{t}\right] d t-\sum_{k=1}^{d} \tilde{Z}_{t}^{k} d W_{t}^{k, \mathbb{Q}}-\sum_{j \in\{B, C\}} \tilde{U}_{t}^{j} d M_{t}^{j, \mathbb{Q}} \\ X_{\tau} & =\left(\hat{V}_{\tau}(\varphi)-\theta_{\tau}\left(\hat{V}, C, I^{T C}, I^{F C}\right)\right),\end{cases}
$$

where $\tilde{U}^{j}, j \in\{B, C\}$, are $\mathbb{G}$-adapted processes in $\mathcal{H}_{\lambda}^{2,2}(\mathbb{Q})$ such that

$$
\sum_{j \in\{B, C\}} \tilde{U}_{t}^{j} d M_{t}^{j, \mathbb{Q}}=-\left(\left(\vartheta_{t}-\overline{X V A}_{t}\right) d J_{t}+\lambda_{t}^{C, \mathbb{Q}}\left(-\theta_{t}^{C}-\overline{X V A}_{t}\right) d t+\lambda_{t}^{B, \mathbb{Q}}\left(\theta_{t}^{B}-\overline{X V A}_{t}\right) d t\right) .
$$

In particular, $X_{t}=X V A_{t}, t \in[0, T]$, where $X V A$ is introduced in Definition 3.9.

Proof. We start from (3.28) and apply the product rule, hence

$$
\begin{aligned}
d X_{t} & =d\left(\overline{X V A}_{t} J_{t}\right)+d\left(1_{\{\tau<T\}} \vartheta_{\tau}\right) \\
& =d \overline{X V A}_{t \wedge \tau}+\overline{X V A}_{t} d J_{t}-\vartheta_{t} d J_{t} .
\end{aligned}
$$

By $(3.22)$ we obtain

$$
\begin{aligned}
d X_{t}= & {\left[f\left(t, \hat{V}-\overline{X V A}, C, I^{T C}, I^{F C}\right)+\left(r_{t}+\lambda_{t}^{C, \mathbb{Q}}+\lambda_{t}^{B, \mathbb{Q}}\right) \overline{X V A_{t}}+\lambda_{t}^{C, \mathbb{Q}} \theta_{t}^{C}-\lambda_{t}^{B, \mathbb{Q}} \theta_{t}^{B}\right] d t } \\
& +\sum_{i=1}^{d} \bar{Z}_{t}^{k} 1_{\{t<\tau\}} d W_{t}^{k, \mathbb{Q}}-\left(\vartheta_{t}-\overline{X V A}_{t}\right) d J_{t} .
\end{aligned}
$$


We note that the process $\sum_{i=1}^{d} \int_{0}^{\cdot} \bar{Z}_{u}^{k} 1_{\{u<\tau\}} d W_{u}^{k, \mathbb{Q}}$ is a $(\mathbb{G}, \mathbb{Q})$-martingale, since $\bar{Z}$ is in $\mathcal{H}^{2, d}(\mathbb{Q})$ due to the immersion hypothesis. From Lemma 5.2.9 in Crépey et al. (2014) we deduce that the process, expressed in differential form

$$
-\left(\left(\vartheta_{t}-\overline{X V A}_{t}\right) d J_{t}+\lambda_{t}^{C, \mathbb{Q}}\left(-\theta_{t}^{C}-\overline{X V A}_{t}\right) d t+\lambda_{t}^{B, \mathbb{Q}}\left(\theta_{t}^{B}-\overline{X V A}_{t}\right) d t\right)
$$

is also a $(\mathbb{G}, \mathbb{Q})$-local martingale. Moreover, we observe that, since $\hat{V} \in \mathcal{S}^{2}(\mathbb{Q})$, also $C \in \mathcal{S}^{2}(\mathbb{Q})$, $C$ being a Lipschitz function of $\hat{V}$. Additionally, the initial margin, be it posted or received, lies in $\mathcal{H}^{2}(\mathbb{Q})$ by assumption. Summing up, both $\theta^{B}$ and $\theta^{C}$, and hence $\vartheta$ belong to the space $\mathcal{H}^{2}(\mathbb{Q})$. On the other hand, $\overline{X V A} \in \mathcal{S}^{2}(\mathbb{Q})$. Recalling that both $\lambda^{C, \mathbb{Q}}$ and $\lambda^{B, \mathbb{Q}}$ are bounded, it follows that the compensated jump term (3.31) is a square integrable martingale. Since the predictable representation property holds in $\mathbb{G}$ with respect to $W^{j, \mathbb{Q}}, M^{j, \mathbb{Q}}, j \in\{B, C\}$, under the measure $\mathbb{Q}$, we obtain that there exists $\tilde{U}^{B}, \tilde{U}^{C} \in \mathcal{H}_{\lambda}^{2,2}(\mathbb{Q})$ satisfying 3.30 . We conclude that the process $X V A$ solves the xVA-BSDE 3.29 under the filtration $\mathbb{G}$.

We can finally combine the solution of the BSDE 3.22 for the clean value with the result of Proposition 3.15 to solve the $\mathbb{G}-B S D E(3.10)$.

Theorem 3.16. Let $V_{t}:=\hat{V}_{t}-X V A_{t}, t \in[0, T]$, on $\{\tau>t\}$, where $\hat{V}$ and $X V A$ are defined in (3.14) and (3.15), respectively. Then, under Assumptions 2.14 and 3.12, the triplet $(V, Z, U) \in$ $\mathcal{S}^{2}(\mathbb{Q}) \times \mathcal{H}^{2, d}(\mathbb{Q}) \times \mathcal{H}_{\lambda}^{2,2}(\mathbb{Q})$ solves the $\mathbb{G}-B S D E(3.10$ with $\mathcal{V}=\hat{V}$, where $Z$ and $U$ are given by

$$
\begin{gathered}
Z_{t}^{k}=\hat{Z}_{t}^{k}-\tilde{Z}_{t}^{k}, k=1, \ldots, d, \\
U_{t}^{j}=-\tilde{U}_{t}^{j}, j \in\{B, C\} .
\end{gathered}
$$

Moreover, the process $V$ satisfies (3.11).

Proof. By Hypothesis 2.1, on $\{t<\tau\}$ we have

$$
\hat{V}_{t}(\varphi)=B_{t}^{r} \mathbb{E}^{\mathbb{Q}}\left[\int_{(t, T]} \frac{d A_{u}}{B_{u}^{r}} \mid \mathcal{F}_{t}\right]=B_{t}^{r} \mathbb{E}^{\mathbb{Q}}\left[\int_{(t, T]} \frac{d A_{u}}{B_{u}^{r}} \mid \mathcal{G}_{t}\right] .
$$

So we consider $\hat{V}$ under $\mathbb{G}$. We also observe that, on $\{t<\tau\}$, we have $\bar{A}_{t}=A_{t}$. Using (3.13) and (3.29), we write the dynamics of $V$ on $\{t<\tau\}$

$$
\begin{aligned}
-d V_{t} & =d \bar{A}_{t}+\left[f\left(t, \hat{V}-X V A, C, I^{T C}, I^{F C}\right) d t-r_{t}\left(\hat{V}_{t}-X V A_{t}\right)\right] d t \\
& -\sum_{k=1}^{d}\left(\hat{Z}_{t}^{k}-\tilde{Z}_{t}^{k}\right) d W_{t}^{k, \mathbb{Q}}-\sum_{j \in\{B, C\}}\left(-\tilde{U}_{t}^{j}\right) d M_{t}^{j, \mathbb{Q}}
\end{aligned}
$$

with terminal condition at $\tau$

$$
V_{\tau}=\hat{V}_{\tau}-X V A_{\tau}=\hat{V}_{\tau}-\left(\theta_{\tau}-\hat{V}_{\tau}\right)=\theta_{\tau} .
$$

Since $Z=\hat{Z}-\tilde{Z} \in \mathcal{H}^{2, d}(\mathbb{Q})$ and $U=-\tilde{U} \in \mathcal{H}_{\lambda}^{2,2}(\mathbb{Q})$, by Theorem 3.5 and Proposition 3.15, we obtain that $(V, Z, U)$ solves the $\mathbb{G}$-BSDE $(3.10)$ and satisfies the required integrability conditions.

Finally, we are now able to prove that 3.11 is equivalent to 3.10 .

Here we assume to work only on $\{\tau>t\}$. Since $V_{t}=\hat{V}-X V A_{t}$ and thanks to Definition 3.9 we have

$$
V_{t}(\varphi)=\hat{V}_{t}(\varphi)+B_{t}^{r} \mathbb{E}^{\mathbb{Q}}\left[1_{\left\{\tau^{C}<\tau^{B}\right\}} \frac{\left(1-R^{C}\right)\left(\hat{V}_{\tau}(\varphi)-C_{\tau-}+I_{\tau-}^{F C}\right)^{-}}{B_{\tau}^{r}}\right.
$$




$$
\begin{aligned}
& -1_{\left\{\tau^{B}<\tau^{C}\right\}} \frac{\left(1-R^{B}\right)\left(\hat{V}_{\tau}(\varphi)-C_{\tau-}-I_{\tau-}^{T C}\right)^{+}}{B_{\tau}^{r}} \\
& -\int_{t}^{\tau} \frac{\left(r_{u}^{f, l}-r_{u}\right)\left(V_{u}(\varphi)-C_{u}-I_{u}^{T C}\right)^{+}-\left(r_{u}^{f, b}-r_{u}\right)\left(V_{u}(\varphi)-C_{u}-I_{u}^{T C}\right)^{-}}{B_{u}^{r}} d u \\
& \left.-\int_{t}^{\tau} \frac{\left(r_{u}^{c, l}-r_{u}\right) C_{u}^{+}-\left(r_{u}^{c, b}-r_{u}\right) C_{u}^{-}}{B_{u}^{r}} d u-\int_{t}^{\tau} \frac{\left(r_{u}^{I, l}-r_{u}\right) I_{u}^{T C}-r_{u}^{I, b} I_{u}^{F C}}{B_{u}^{r}} d u \mid \mathcal{G}_{t}\right] .
\end{aligned}
$$

By (3.9c) we obtain

$$
\begin{aligned}
V_{t}(\varphi)=\hat{V}_{t}(\varphi) & +B_{t}^{r} \mathbb{E}^{\mathbb{Q}}\left[1_{\left\{\tau^{C}<\tau^{B}\right\}} \frac{\left(1-R^{C}\right)\left(\hat{V}_{\tau}(\varphi)-C_{\tau-}+I_{\tau-}^{F C}\right)^{-}}{B_{\tau}^{r}}\right. \\
& -1_{\left\{\tau^{B}<\tau^{C}\right\}} \frac{\left(1-R^{B}\right)\left(\hat{V}_{\tau}(\varphi)-C_{\tau-}-I_{\tau-}^{T C}\right)^{+}}{B_{\tau}^{r}} \\
& \left.+\int_{t}^{\tau} \frac{f\left(u, V, C, I^{T C}, I^{F C}\right)}{B_{u}^{r}} d u \mid \mathcal{G}_{t}\right] .
\end{aligned}
$$

Assumption 3.8 and 2.8 ensure that

$$
V_{t}(\varphi)=\hat{V}_{t}(\varphi)+B_{t}^{r} \mathbb{E}^{\mathbb{Q}}\left[\int_{t}^{\tau} \frac{f\left(u, V, C, I^{T C}, I^{F C}\right)}{B_{u}^{r}} d u+\frac{\theta_{\tau}\left(\hat{V}(\varphi), C, I^{T C}, I^{F C}\right)-\hat{V}_{\tau}(\varphi)}{B_{\tau}^{r}} \mid \mathcal{G}_{t}\right] .
$$

Now, we apply (3.14), the tower property and Hypothesis 2.1, so that

$$
\begin{aligned}
V_{t}(\varphi) & =B_{t}^{r} \mathbb{E}^{\mathbb{Q}}\left[\int_{t}^{\tau} \frac{f\left(u, V, C, I^{T C}, I^{F C}\right)}{B_{u}^{r}} d u+1_{\{\tau \leq T\}} \frac{\theta_{\tau}\left(\hat{V}(\varphi), C, I^{T C}, I^{F C}\right)}{B_{\tau}^{r}} \mid \mathcal{G}_{t}\right] \\
& +B_{t}^{r} \mathbb{E}^{\mathbb{Q}}\left[\int_{(t, T]} \frac{d A_{u}}{B_{u}^{r}}-\int_{(t, T]} \frac{d A_{u}}{B_{\tau}^{r}} \mid \mathcal{G}_{t}\right] .
\end{aligned}
$$

Finally, again by (2.8), we have

$$
V_{t}(\varphi)=B_{t}^{r} \mathbb{E}^{\mathbb{Q}}\left[\int_{(t, T]} \frac{d \bar{A}_{u}}{B_{u}^{r}}+\int_{t}^{\tau} \frac{f\left(u, V, C, I^{T C}, I^{F C}\right)}{B_{u}^{r}} d u+\frac{\theta_{\tau}\left(\hat{V}(\varphi), C, I^{T C}, I^{F C}\right)}{B_{\tau}^{r}} \mid \mathcal{G}_{t}\right] .
$$

We now provide an explicit formula for the value adjustments under the filtration $\mathbb{F}$. This representation is particularly useful from a computational point of view: risk factors can be simulated under the smaller filtration $\mathbb{F}$ and the computation of value adjustments does not require the simulation of default times. It is an immediate consequence of Proposition 3.14 .

Corollary 3.17. Let $(\overline{X V A}, \bar{Z})$ be the unique solution to the pre-default $x V A-B S D E$ under $\mathbb{F}$ (3.22). Define the process $\tilde{r}=\left(\tilde{r}_{t}\right)_{t \in[0, T]}$ by setting $\tilde{r}:=r+\lambda^{C, \mathbb{Q}}+\lambda^{B, \mathbb{Q}}$. Under Assumptions 2.14 and 3.12 the stochastic process $\overline{X V A}$ admits the following representation.

$$
\overline{X V A}_{t}=-\overline{C V A}_{t}+\overline{D V A}_{t}+\overline{F V A}_{t}+\overline{C o l V A}_{t}+\overline{M V A}_{t}
$$

where

$$
\overline{C V A}_{t}:=B_{t}^{\tilde{r}} \mathbb{E}^{\mathbb{Q}}\left[\left(1-R^{C}\right) \int_{t}^{T} \frac{1}{B_{u}^{\tilde{r}}}\left(\hat{V}_{u}(\varphi)-C_{u}+I_{u}^{F C}\right)^{-} \lambda_{u}^{C, \mathbb{Q}} d u \mid \mathcal{F}_{t}\right]
$$




$$
\begin{aligned}
\overline{D V A}_{t} & :=B_{t}^{\tilde{r}} \mathbb{E}^{\mathbb{Q}}\left[\left(1-R^{B}\right) \int_{t}^{T} \frac{1}{B_{u}^{\tilde{r}}}\left(\hat{V}_{u}(\varphi)-C_{u}-I_{u}^{T C}\right)^{+} \lambda_{u}^{B, \mathbb{Q}} d u \mid \mathcal{F}_{t}\right], \\
\overline{F V A}_{t} & :=B_{t}^{\tilde{r}} \mathbb{E}^{\mathbb{Q}}\left[\int_{t}^{T} \frac{\left(r_{u}^{f, l}-r_{u}\right)\left(V_{u}(\varphi)-C_{u}-I_{u}^{T C}\right)^{+}-\left(r_{u}^{f, b}-r_{u}\right)\left(V_{u}(\varphi)-C_{u}-I_{u}^{T C}\right)^{-}}{B_{u}^{\tilde{r}}} d u \mid \mathcal{F}_{t}\right], \\
\overline{\operatorname{ColVA}}_{t} & :=B_{t}^{\tilde{r}} \mathbb{E}^{\mathbb{Q}}\left[\int_{t}^{T} \frac{\left(r_{u}^{c, l}-r_{u}\right) C_{u}^{+}-\left(r_{u}^{c, b}-r_{u}\right) C_{u}^{-}}{B_{u}^{\tilde{r}}} d u \mid \mathcal{F}_{t}\right], \\
\overline{M V A}_{t} & :=B_{t}^{\tilde{r}} \mathbb{E}^{\mathbb{Q}}\left[\int_{t}^{T} \frac{\left(r_{u}^{I, l}-r_{u}\right) I_{u}^{T C}-r_{u}^{I, b} I_{u}^{F C}}{B_{u}^{\tilde{r}}} d u \mid \mathcal{F}_{t}\right] .
\end{aligned}
$$

In the literature there has been an intense debate regarding the problem given by the possible overlap between FVA and DVA, see e.g. Hull and White (2012), Andersen et al. (2019), Brigo et al. (2019) and references therein. This problem, however, is due to accounting inconsistencies that do not affect our pricing equations. We limit ourselves to mention that a sound treatment of the issue is provided by Brigo et al. (2019) and that their solution can be embedded in our framework at the cost of further notations.

3.3. The xVA-CSA consistency problem. We here provide an arbitrage-free framework for the CSA discounting practice, i.e. the practice of using contingent claim specific discounting regimes.

In Section 3.1 we assumed that the clean value refers to an idealized fully collateralized transaction where the collateral rate is simply $r$. The situation in practice is more complicated. The market practice adopted for the computation of clean prices involves a multitude of discount curves. Possible examples from the current market practice are

- The (clean) value of a perfectly uncollateralized derivative might be discounted by a bank by means of a bank-specific funding curve with associated short rate $r^{f}$ (this could correspond to the Libor rate for a bank belonging to the Libor panel), see e.g. Piterbarg (2010).

- The (clean) value of a derivative collateralized in a foreign currency is discounted on the market at a rate depending on cross currency bases, see the formulas and derivations in Gnoatto and Seiffert 2020).

It is quite natural to ask why banks employ many discount regimes for clean values and, on top of that, $\mathrm{xVA}$ corrections. The main reason is purely pragmatic and non-mathematical: from the perspective of a trading desk it is convenient to treat CSAs by means of different discount regimes, because this allows to deal with portfolio market risk via traditional trading-desk techniques, such as curve trades (i.e. e.g. buying/selling interest rate swaps on different buckets/maturities along the curve). Hedging the expectation of an integral such as the FVA term in practice is much more complicated. A possible approximate treatment involves discretizing the time integral and treating the resulting Riemann sum over time as a portfolio of claims. In view of the aforementioned difficulty, market operators prefer to obtain an additive price representation, where discount curves are used to reduce the magnitude of the (funding related) xVA terms, which are more difficult to hedge.

From now on, we shall assume that the bank has two internal desks, dubbed the front-office desk and the $x V A$ desk, respectively. The front-office desk is responsible for the calculation of clean values and for the trading activity required to hedge market risk of the clean values. The xVA desk instead computes and hedges all the value adjustments and is forced, according to internal rules of the bank, to adopt for each transaction the clean value dictated by the front-office desk. The fact that the xVA desk is a clean-value-taker implies that care is needed when computing xVAs, in order to avoid double counting effects. 
The xVA desk has to deal with two different clean values for the same transaction. On the one side, it performs an arbitrage-free pricing by computing the clean value by means of the $\mathbb{F}$-BSDE (3.13). On the other side, it has to use the clean value prescribed by the front office function, which constitutes the official clean value accepted within the bank. The xVA desk is then faced with the following challenge:

Problem 3.18 (xVA-CSA consistency problem). Produce a price decomposition of $V$ in terms of clean value and $x V A$ such that

(i) the representation of $V$ is coherent with the $\mathbb{G}-B S D E(3.10)$, and

(ii) the clean price corresponds to the one prescribed by the front-office function.

We now provide a solution for Problem 3.18 by using the results of Section 3 .

Remark 3.19. To provide a concrete example, consider the situation where the trading desk of the bank enters into two perfectly collateralized transactions with two different counterparties, the first one being e.g. a clearing house such as LCH, the other one being another clearing house such as Eurex. Although the dividend process of the claim is the same for both transactions, the collateral remuneration provided by the trade with Eurex and the trade with LCH is different. The spread in the collateral remuneration between EUREX and LCH is called Eurex-LCH basis, see e.g. Mackenzie Smith (2017) for a more detailed discussion. This will result in the two clean values being computed by means of different discounting rates.

In summary, the market practice of discounting cashflows according to trade-specific collateral rates implies that, within the bank, a single transaction will be discounted at least according to two different regimes. Initially, the front-office determines the clean value by discounting cash flows through an ideal market collateral rate $\hat{r}$. Hence the front-office clean value $\hat{\mathcal{P}}=\left(\hat{\mathcal{P}}_{t}\right)_{t \in[0, T]}$ is obtained from the $\mathbb{F}$-BSDE

$$
\left\{\begin{array}{l}
-d \hat{\mathcal{P}}_{t}=-\sum_{k=1}^{d} \hat{Z}_{t}^{*, k} d W_{t}^{k, \mathbb{Q}}+d A_{t}-\hat{r} \hat{\mathcal{P}}_{t} d t, \\
\hat{\mathcal{P}}_{T}=0 .
\end{array}\right.
$$

On the other side, the xVA desk first computes the clean value $\hat{V}=\left(\hat{V}_{t}\right)_{t \in[0, T]}$ as the solution to the $\mathbb{F}$-BSDE (3.13), i.e. by solving

$$
\left\{\begin{array}{l}
-d \hat{V}_{t}=-\sum_{k=1}^{d} \hat{Z}_{t}^{k} d W_{t}^{k, \mathbb{Q}}+d A_{t}-r_{t} \hat{V}_{t} d t, \\
\hat{V}_{T}=0
\end{array}\right.
$$

From a valuation perspective, if clean values represented the prices of real transactions, the presence of different discounting rules would immediately imply the presence of trivial arbitrage opportunities in the market. Only the endogenous price $\hat{V}$ in $(3.36)$ is compatible with the arbitrage-free setting of Section 3. On the other hand, the xVA desk is forced to provide results in terms of the discounting regimes imposed by the front-office. The two approaches can be combined in an arbitrage-free setting by means of the following invariance property of linear BSDEs.

Lemma 3.20. Let $\left(\hat{V}, \hat{Z}^{1}, \ldots, \hat{Z}^{d}\right)$ be the unique solution of the $\mathbb{F}$-BSDE (3.36). Under Assumption 3.4 for $A$, the value process $\hat{V}$ admits the two equivalent representations

i) $x V A$-discounting representation

$$
\hat{V}_{t}=B_{t}^{r} \mathbb{E}^{\mathbb{Q}}\left[\int_{(t, T]} \frac{d A_{t}}{B_{u}^{r}} \mid \mathcal{F}_{t}\right],
$$


ii) CSA-discounting representation

$$
\hat{V}_{t}=\hat{\mathcal{P}}_{t}-\operatorname{DiscV} A_{t}
$$

where DiscV $A_{t}$ represents the discounting valuation adjustment, defined as

$$
\operatorname{DiscV} A_{t}:=B_{t}^{\hat{r}} \mathbb{E}^{\mathbb{Q}}\left[\int_{t}^{T}\left(r_{u}-\hat{r}\right) \frac{\hat{V}_{u}}{B_{u}^{\hat{r}}} d u \mid \mathcal{F}_{t}\right],
$$

and $\hat{\mathcal{P}}$ is the value process in the solution $\left(\hat{\mathcal{P}}, \hat{Z}^{*, 1}, \ldots, \hat{Z}^{*, d}\right)$ of the $\mathbb{F}$-BSDE 3.35

$$
\hat{\mathcal{P}}_{t}=B_{t}^{\hat{r}} \mathbb{E}^{\mathbb{Q}}\left[\int_{(t, T]} \frac{d A_{t}}{B_{u}^{\hat{r}}} \mid \mathcal{F}_{t}\right] \text {. }
$$

Proof. The integral representation (3.37) is immediate. To obtain (3.38) we rewrite the $\mathbb{F}$-BSDE (3.36) adding and subtracting the term $\hat{r} \hat{V}_{t}$, i.e.,

$$
\left\{\begin{array}{l}
-d \hat{V}_{t}=-\sum_{k=1}^{d} \hat{Z}_{t}^{k} d W_{t}^{k, \mathbb{Q}}+d A_{t}-\left(r_{t}-\hat{r}_{t}\right) \hat{V}_{t} d t-\hat{r}_{t} \hat{V}_{t} d t \\
\hat{V}_{T}=0 .
\end{array}\right.
$$

The value process of the solution is given by

$$
\hat{V}_{t}=B_{t}^{\hat{r}} \mathbb{E}^{\mathbb{Q}}\left[\int_{(t, T]} \frac{d A_{t}}{B_{u}^{\hat{r}}} \mid \mathcal{F}_{t}\right]-B_{t}^{\hat{r}} \mathbb{E}^{\mathbb{Q}}\left[\int_{t}^{T}\left(r_{u}-\hat{r}_{u}\right) \frac{\hat{V}_{u}}{B_{u}^{\hat{r}}} d u \mid \mathcal{F}_{t}\right],
$$

where we recognize the first expectation as $\hat{P}$, whereas the second one provides $\operatorname{DiscV} A$.

The $\mathbb{G}$-BSDE for the dynamics of $V$ under $\mathbb{Q}$ can be written as

$$
\left\{\begin{aligned}
-d V_{t}(\varphi)= & d \bar{A}_{t}+\left(f\left(t, V, C, I^{T C}, I^{F C}\right)-r_{t} V_{t}(\varphi)\right) d t \\
& -\sum_{k=1}^{d} Z_{t}^{k} d W_{t}^{k, \mathbb{Q}}-\sum_{j \in\{B, C\}} U_{t}^{j} d M_{t}^{j, \mathbb{Q}} \\
V_{\tau}(\varphi)= & \theta_{\tau}(\hat{V}, C, I)
\end{aligned}\right.
$$

for $t \leq \tau$, where $\bar{A}_{t}$ is defined in (2.8), $Z_{t}=\left(Z_{t}^{1}, \ldots, Z_{t}^{d}\right)$, and $U_{t}=\left(U_{t}^{B}, U_{t}^{C}\right)$, represent the control processes given by $\mathbb{G}$-predictable processes, and $f\left(t, V, C, I^{T C}, I^{F C}\right)$ is the $\mathbb{G}$-BSDE driver given by (3.9c). The close-out condition is

$$
\begin{aligned}
V_{\tau}(\varphi) & =\hat{\mathcal{P}}_{\tau}+1_{\left\{\tau^{C}<\tau^{B}\right\}}\left(1-R^{C}\right)\left(\hat{\mathcal{P}}_{\tau}-C_{\tau-}+I_{\tau-}^{F C}-\operatorname{DiscV} A_{\tau}\right)^{-} \\
& -1_{\left\{\tau^{B}<\tau^{C}\right\}}\left(1-R^{B}\right)\left(\hat{\mathcal{P}}_{\tau}-C_{\tau-}-I_{\tau-}^{T C}-\operatorname{DiscV} A_{\tau}\right)^{+} .
\end{aligned}
$$

By using the same arguments given for Theorem 3.16 and taking into account Definition 3.9, we obtain the following result, with the help of Lemma 3.20 .

Proposition 3.21. Under Assumption 3.4 and 3.12, the $\mathbb{G}-B S D E$ (3.41) admits a unique solution $(V, Z, U) \in \mathcal{S}^{2}(\mathbb{Q}) \times \mathcal{H}^{2, d}(\mathbb{Q}) \times \mathcal{H}_{\lambda}^{2,2}(\mathbb{Q})$ with

$$
V_{t}(\varphi)=B_{t}^{\hat{r}} \mathbb{E}^{\mathbb{Q}}\left[\int_{(t, T]} \frac{d A_{u}}{B_{u}^{\hat{r}}} \mid \mathcal{F}_{t}\right]-X V A_{t}=\hat{\mathcal{P}}_{t}-\widehat{X V A}_{t}
$$

for $t<\tau$, where

$$
\widehat{X V A}:=X V A+\operatorname{Disc} V A
$$


with

$$
\begin{aligned}
X V A_{t} & :=F V A_{t}+\operatorname{ColV} A_{t}+M V A_{t}-C V A_{t}+D V A_{t} \\
& =F V A_{t}+\operatorname{Col} V A_{t}+M V A_{t} \\
& -B_{t}^{r} \mathbb{E}^{\mathbb{Q}}\left[1_{\{\tau<T\}} 1_{\left\{\tau^{C}<\tau^{B}\right\}}\left(1-R^{C}\right) \frac{1}{B_{\tau}^{r}}\left(\hat{\mathcal{P}}_{\tau}-C_{\tau-}+I_{\tau-}^{F C}-\operatorname{DiscV} A_{\tau}\right)^{-} \mid \mathcal{G}_{t}\right] \\
& +B_{t}^{r} \mathbb{E}^{\mathbb{Q}}\left[1_{\{\tau<T\}} 1_{\left\{\tau^{B}<\tau^{C}\right\}}\left(1-R^{B}\right) \frac{1}{B_{\tau}^{r}}\left(\hat{\mathcal{P}}_{\tau}-C_{\tau-}+I_{\tau-}^{T C}-\operatorname{DiscV} A_{\tau}\right)^{+} \mid \mathcal{G}_{t}\right]
\end{aligned}
$$

and

$$
\operatorname{DiscV} A_{t}:=B_{t}^{\hat{r}} \mathbb{E}^{\mathbb{Q}}\left[\int_{t}^{T}\left(r_{u}-\hat{r}_{u}\right) \frac{\hat{V}_{u}}{B_{u}^{\hat{r}}} d u \mid \mathcal{F}_{t}\right],
$$

where FVA, ColVA, MVA are defined in line with Definition 3.9.

Proof. Existence and uniqueness for the solution of (3.41) follow along the lines of Section 3.

The impact of CSA discounting on derivative exposures is represented by the presence of $\hat{\mathcal{P}}$ in the CVA and DVA terms in 3.45. Furthermore, decomposition 3.45 shows the exposure profile in the portfolio under $r$ or $\hat{r}$, respectively.

3.4. xVA multiple aggregation level. In this section we provide a $\mathbb{G}$-BSDE for the global portfolio of the bank given by the aggregation of several sub-portfolios of trades. Current market practice shows that the set of trades between the bank and the counterparty can be typically split into several subsets reflecting multiple aggregation levels. One can distinguish between funding/margin sets and netting sets. A margin (or funding) set $\mathcal{M}$ is a set of claims whose aggregated clean values (exposures) are fully or partially covered by a CSA (collateral agreement). We denote by $N_{\mathcal{M}}$ the number of margin sets in a portfolio $\mathcal{A}$. A netting set $\mathcal{N}$ is a set of margin sets whose post-margin exposures can be aggregated. We denote by $N_{\mathcal{N}}$ the number of netting sets in a portfolio $\mathcal{A}$.

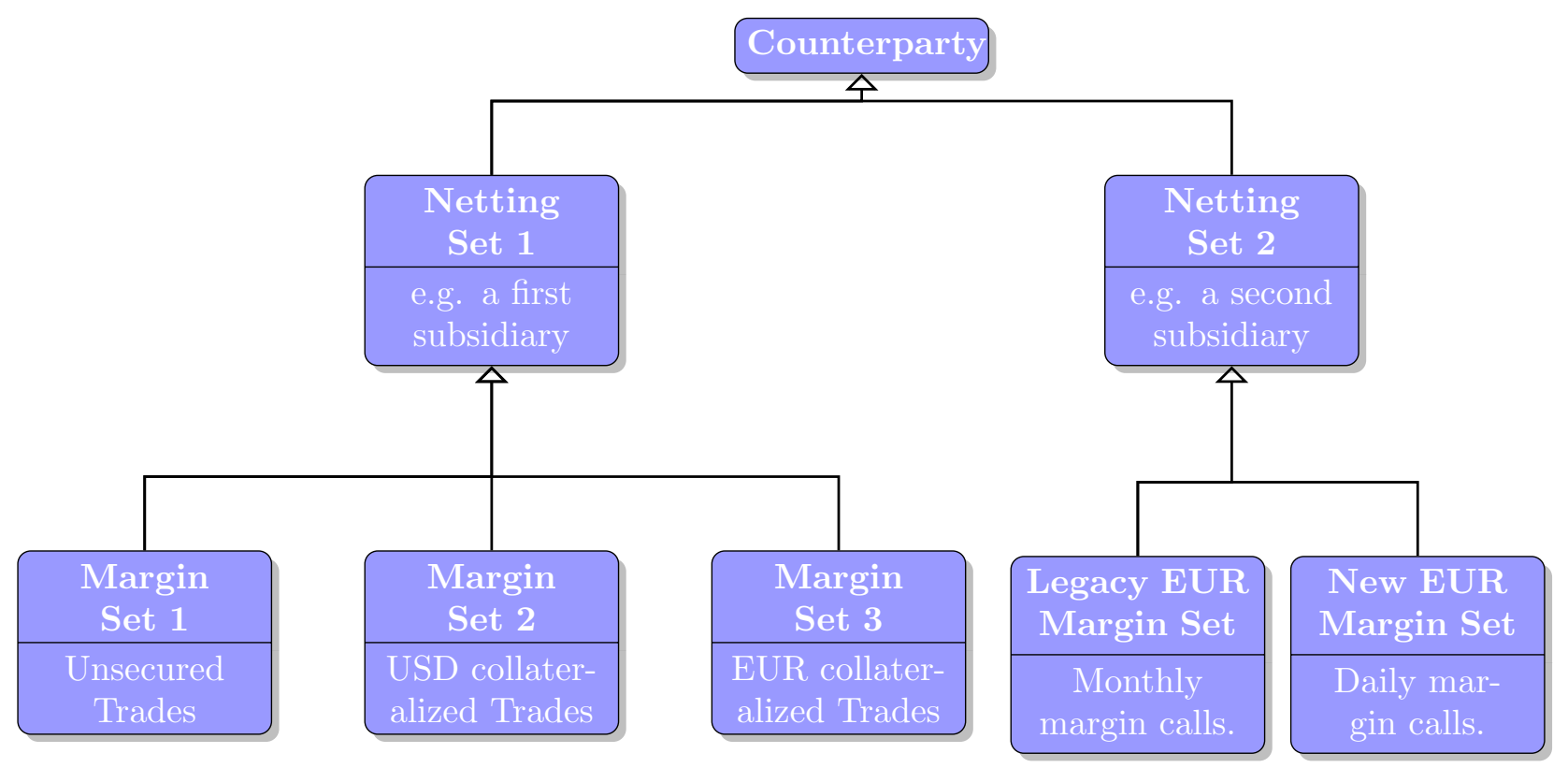

FIgURE 1. A possible hierarchical structure of aggregation levels. 
Example 3.22. Funding/margin sets are traded between the bank and the counterparty that share the same funding policy. This corresponds to different CSAs: for example, one CSA (Margin Set 2) could group all trades for which collateral is exchanged in USD (e.g. for foreign exchange derivatives), whereas another CSA (Margin Set 3) could be relevant for all instruments collateralized in EUR. Finally, trades that are not collateralized, but whose exposures are netted among each other, can be also grouped in a separate margin/funding set, corresponding to Margin Set 1 in Figure 1 .

The protection provided by collateralization agreements might however be imperfect, hence a legal agreement between the bank and the counterparty might allow for the netting of residual post collateral exposures arising from different margin sets. This corresponds to Netting Set 1 in Figure 1. Another typical source of multiple aggregation levels is the historical stratification of legal agreements: in Figure 1 we have a second netting set, corresponding to a second subsidiary of the parent counterparty, where legacy trades are covered by an old CSA agreement involving monthly margin calls, whereas all trades entered after a certain date are covered by a newer CSA agreement involving daily margin calls.

Remark 3.23. A further level of complexity could arise when the parent and the subsidiaries have different default times: this introduces further complications when modeling the close-out condition because one might have e.g. a situation where the default of a subsidiary is covered by the parent. Such issues are left for future research. From a practical point of view it is also difficult to find calibration instruments for default probabilities, since subsidiaries typically do not enjoy a liquid CDS market.

We assume that the portfolio $\mathcal{A}$ of trades between the bank and the counterparty consists of $K$ trades, that we identify by means of the respective payment processes, i.e., $\mathcal{A}=\left\{A^{1}, \ldots A^{K}\right\}$. We use again $\hat{V}^{m}$ to denote the clean reference value of the claims $A^{m}, m=1, \ldots K$, representing also their credit exposure before collateral is applied.

For every claim $A^{m} \in \mathcal{A}, m=1, \ldots K$, we assume that the margin set for initial and variation margin coincide.

The structure of the portfolio is illustrated in Figure 1, where the first row depicts the composition of all margin sets as groups of claims and the second line describes the netting sets as groups of margin sets. Hence,

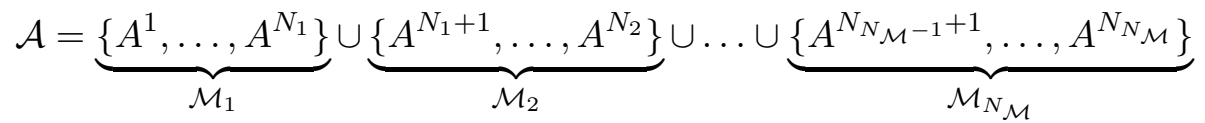

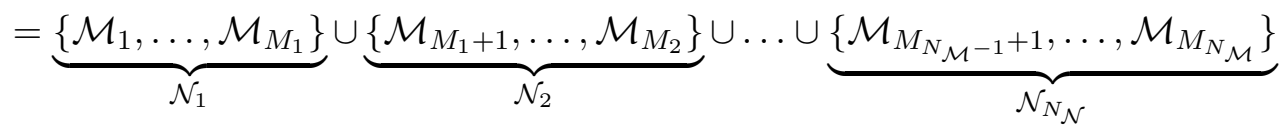

where we have $N_{N_{\mathcal{M}}}=K$.

We now provide a $\mathbb{G}$-BSDE for the portfolio at the $m_{2}$-th netting set-level in $(3.46)$ by using the results of Section 3.1. The presence of different margin sets is represented by the introduction of different collateral accounts. Multiple netting sets are accounted for by summing value adjustments over all netting sets, each netting set possibly featuring several margin sets.

To this purpose, we resort to a multi-index notation for the processes involved. In particular, we denote by $\bar{A}_{t}^{m, m_{1}, m_{2}}$ the $m$-th contingent claim in the portfolio $\mathcal{A}$ belonging to the $m_{1}$-th netting setlevel and at the $m_{2}$-th margin set-level. The same notation applies to the control processes $Z_{t}^{k, m_{1}, m_{2}}$ and $U_{t}^{j, m_{1}, m_{2}}$, for $k=1, \ldots, d$ and $j \in\{B, C\}$. Hence, the value process $V_{t}^{m_{2}}$ corresponding to the 
$m_{2}$-th netting set, $m_{2}=1, \ldots, N_{\mathcal{N}}$, satisfies the following $m_{2}$-th netting set-level BSDE

$$
\begin{aligned}
& -d V_{t}^{m_{2}}(\varphi)=\sum_{m_{1}=1}^{\left|\mathcal{N}_{m_{2}}\right|\left|\mathcal{M}_{m_{1}}\right|} \sum_{m=1} d \bar{A}_{t}^{m, m_{1}, m_{2}} \\
& +\left[f\left(t, V^{m_{2}},\left(C^{\mathcal{M}_{m_{1}}, \mathcal{N}_{m_{2}}}\right)_{m_{1}=1, \ldots,\left|\mathcal{N}_{m_{2}}\right|},\left(I^{\mathcal{M}_{m_{1}}, \mathcal{N}_{m_{2}}}\right)_{m_{1}=1, \ldots,\left|\mathcal{N}_{m_{2}}\right|}\right)-r_{t} V_{t}^{m_{2}}(\varphi)\right] d t \\
& -\sum_{m_{1}=1}^{\left|\mathcal{N}_{m_{2}}\right|} \sum_{m=1}^{\left|\mathcal{M}_{m_{1}}\right|} \sum_{k=1}^{d} Z_{t}^{k, m_{1}, m_{2}} d W_{t}^{k, \mathbb{Q}}-\sum_{m_{1}=1}^{\left|\mathcal{N}_{m_{2}}\right|} \sum_{m=1}^{\left|\mathcal{M}_{m_{1}}\right|} \sum_{j \in\{B, C\}} U_{t}^{j, m_{1}, m_{2}} d M_{t}^{j, \mathbb{Q}},
\end{aligned}
$$

for $m_{2}=1, \ldots, N_{\mathcal{N}}$.

By applying Theorem 3.21, we obtain the following

Proposition 3.24. There exists a unique solution $\left(V^{m_{2}}, Z^{m_{1}, m_{2}}, U^{m_{1}, m_{2}}\right) \in \mathcal{S}^{2}(\mathbb{Q}) \times \mathcal{H}^{2, d}(\mathbb{Q}) \times \mathcal{H}_{\lambda}^{2,2}(\mathbb{Q})$ to the $\mathbb{G}-B S D E$ (3.47) with close-out condition

$$
\begin{aligned}
V_{\tau}^{m_{2}}(\varphi) & =\theta_{\tau}^{m_{2}}=\sum_{m_{1}=1}^{\left|\mathcal{N}_{m_{2}}\right|} \sum_{m=1}^{\left|\mathcal{M}_{m_{1}}\right|}\left(\hat{\mathcal{P}}_{\tau}^{m, m_{1}, m_{2}}-\operatorname{DiscV} A_{\tau}^{m, m_{1}, m_{2}}\right) \\
& +1_{\tau^{C}<\tau^{B}}\left(1-R^{C}\right)\left(\sum_{m_{1}=1}^{\left|\mathcal{N}_{m_{2}}\right|} \sum_{m=1}^{\left|\mathcal{M}_{m_{1}}\right|}\left(\hat{\mathcal{P}}_{\tau}^{m, m_{1}, m_{2}}-\operatorname{DiscV} A_{\tau}^{m, m_{1}, m_{2}}\right)-C_{\tau-}^{\mathcal{M}_{m_{1}}, \mathcal{N}_{m_{2}}}-I_{\tau-}^{T C, \mathcal{M}_{m_{1}}, \mathcal{N}_{m_{2}}}\right)^{-}
\end{aligned}
$$

$$
-1_{\tau^{B}<\tau^{C}}\left(1-R^{B}\right)\left(\sum_{m_{1}=1}^{\left|\mathcal{N}_{m_{2}}\right|} \sum_{m=1}^{\left|\mathcal{M}_{m_{1}}\right|}\left(\hat{\mathcal{P}}_{\tau}^{m, m_{1}, m_{2}}-\operatorname{DiscV} A_{\tau}^{m, m_{1}, m_{2}}\right)-C_{\tau-}^{\mathcal{M}_{m_{1}}, \mathcal{N}_{m_{2}}}-I_{\tau-}^{F C, \mathcal{M}_{m_{1}}, \mathcal{N}_{m_{2}}}\right)^{+} .
$$

Remark 3.25. It is easy to see that the value $\mathscr{V}^{K}(\varphi)$ of a portfolio consisting of $K$ claims is given by

$$
\mathscr{V}_{t}^{K}(\varphi)=\sum_{m=1}^{K} \hat{\mathcal{P}}_{t}^{m}-\widehat{X V A}_{t}^{K}, t \leq \tau,
$$

where each $\mathcal{P}^{m}, m=1, \ldots, K$, satisfies a BSDE of the form (3.35), while

$$
\widehat{X V A}_{t}^{K}:=X V A_{t}^{K}+\sum_{m_{2}=1}^{N_{\mathcal{N}}} \sum_{m_{1}=1}^{\left|\mathcal{N}_{m_{2}}\right|} \sum_{m=1}^{\left|\mathcal{M}_{m_{1}}\right|} \operatorname{DiscV} A_{t}^{m, m_{1}, m_{2}}
$$

and

$$
X V A_{t}^{K}:=F V A_{t}^{K}+\operatorname{Col} V A_{t}^{K}+M V A_{t}^{K}-C V A_{t}^{K}+D V A_{t}^{K},
$$

for $t \leq \tau$. We further observe that each $X V A^{K}$ in 3.51 is of the form $\sum_{m_{2}=1}^{N_{\mathcal{N}}} X V A^{m_{2}}$, where $X V A^{m_{2}}$ are the valuation adjustments appearing in the solution to the $\mathbb{G}$-BSDE (3.47).

3.5. Incremental xVA charge. The $\mathbb{G}$-BSDE (3.47) encompasses two levels of complexity. The first is the presence of several sub-portfolios, which we have discussed above. Secondly, the presence of bid-offer spread in the rates, coupled with the recursive nature of the FVA terms implies that the XVA of a portfolio of $K$ trades does not coincide with the sum of the XVAs of the single $K$ trades. This determines non-linearity effects in the xVA valuation as we describe below.

Let us assume now that the counterparty wishes to enter into a further $(K+1)$-th trade with the bank. If entered, the newly introduced $(K+1)$-th claim would contribute to the global riskiness of the portfolio between the bank and the counterparty. It is natural to ask then what is the price the bank should charge to the newly introduced $(K+1)$-th claim given the presence of the already existing $K$ 
claims. The current market practice involves the computation of an incremental $x V A$ charge, where two different scenarios are compared.

(i) Base scenario: The value of the portfolio is given by $\mathscr{V}^{K}(\varphi)$ as in formula (3.49). This corresponds to the value of the portfolio before the inclusion of the candidate new trade.

(ii) Full scenario: The value of the portfolio is given by $\mathscr{V}^{K+1}(\varphi)$, computed in line with formula (3.49). This corresponds to the value of the portfolio after the inclusion of the candidate $(K+1)$-th contingent claim.

The bank determines the price to be charged to the counterparty as the difference between the value of the portfolio under the full and the base scenario given by the incremental value

$$
\Delta V_{t}^{K+1}(\varphi):=\mathscr{V}_{t}^{K+1}(\varphi)-\mathscr{V}_{t}^{K}(\varphi) .
$$

We can isolate the impact of the $(K+1)$-th trade by considering

$$
\begin{aligned}
\Delta V_{t}^{K+1}(\varphi) & =\mathscr{V}_{t}^{K+1}(\varphi)-\mathscr{V}_{t}^{K}(\varphi) \\
& =\sum_{m=1}^{K+1} \hat{\mathcal{P}}_{t}^{m}-\widehat{X V A}_{t}^{K+1}-\sum_{m=1}^{K} \hat{\mathcal{P}}_{t}^{m}+\widehat{X V A}_{t}^{K} \\
& =\hat{\mathcal{P}}_{t}^{K+1}-\left(X V A_{t}^{K+1}-X V A_{t}^{K}\right)-\operatorname{DiscV} A_{t}^{K+1} \\
& =\hat{\mathcal{P}}_{t}^{K+1}-\Delta X V A_{t}-\operatorname{DiscV} A_{t}^{K+1}
\end{aligned}
$$

for $t \leq \tau$, where, in the last step, we implicitly defined the incremental $x V A$ charge

$$
\Delta X V A_{t}:=X V A_{t}^{K+1}-X V A_{t}^{K}
$$

as the adjustment to be charged on the $(K+1)$-th claim, given the presence of the already existing $K$ claims in the portfolio. We obtain the non-linearity effect defined as

$$
N L_{t}\left(V^{K+1}\right):=V_{t}^{K+1}(\varphi)-\Delta V_{t}^{K+1}(\varphi), t \leq \tau,
$$

where $V^{K+1}$ is the value of the portfolio consisting only of the $(K+1)$-th claim and $\Delta V_{t}^{K+1}(\varphi)$ is the incremental charge as defined in (3.52). The non-linearity effect coincides with the difference of the incremental xVA charge and the xVA associated only to the $(K+1)$-th claim, in fact

$$
\begin{aligned}
N L_{t}\left(V^{K+1}\right) & =V_{t}^{K+1}(\varphi)-\Delta V_{t}^{K+1}(\varphi) \\
& =\left(\hat{P}_{t}^{K+1}-X V A_{t}-\operatorname{DiscV} A_{t}^{K+1}\right)-\left(\hat{P}_{t}^{K+1}-\Delta X V A_{t}-\operatorname{DiscV} A_{t}^{K+1}\right) \\
& =\Delta X V A_{t}-X V A_{t} .
\end{aligned}
$$

In the present setting the clean valuation of the contingent claim is still linear, hence the clean value of the portfolio still corresponds to the sum of the clean values of the single claims,but in general $\triangle X V A_{t}-X V A_{t} \neq 0$. The stand-alone $\mathrm{xVA}$ of the $(K+1)$-th claim is higher than $\triangle X V A$. Moreover, $N L_{t}\left(V^{K+1}\right)=0$ only when there are no portfolio/netting effects.

\section{EXAMPLE AND NUMERICAL ILLUSTRATION}

We conclude the paper by presenting an example using a lognormal model for a single risky asset. Under the setting and assumptions of the previous sections we consider a single risky asset $S=$ $\left(S_{t}\right)_{t \in[0, T]}$ that pays dividends at a rate $\kappa=\left(\kappa_{t}\right)_{t \in[0, T]}$, with dividend process $D_{t}=\int_{0}^{t} \kappa_{s} S_{s} d s, t \in$ $[0, T]$. 
Under the martingale measure $\mathbb{Q}$ the risky asset evolves according to

$$
d S_{t}=S_{t}\left(\left(r_{t}^{r}-\kappa_{t}\right) d t+\sigma_{t} d W_{t}^{\mathbb{Q}}\right)
$$

where $r^{r}=\left(r_{t}^{r}\right)_{t \in[0, T]}$ is the repo rate associated to the asset $S$. We now consider a simple contingent claim, namely a forward written on the asset $S$. The dividend process of the claim $A^{1}=\left(A_{t}^{1}\right)_{t \in[0, T]}$, is given by

$$
A_{t}^{1}=1_{\{t=T\}}\left(S_{T}-K^{1}\right),
$$

for $K^{1}$ a positive constant. We recall that the clean value $\hat{V}$ satisfying (3.13) represents a fictious value process for the claim under the assumption of a perfect collateralization scheme that annihilates counterparty risk, see Assumption 3.4 .

According to Theorem 3.6 the arbitrage free price of the forward is

$$
\hat{V}_{t}^{1}(\varphi)=\mathbb{E}^{\mathbb{Q}}\left[B_{t}^{r} \int_{(t, T]} \frac{d A_{u}^{1}}{B_{u}^{r}} \mid \mathcal{F}_{t}\right]=B_{t}^{r} \mathbb{E}^{\mathbb{Q}}\left[\frac{S_{T}-K^{1}}{B_{T}^{r}} \mid \mathcal{F}_{t}\right] .
$$

Assume now that the bank enters a forward with a counterparty without any collateral agreement and without any previous existing trade: there is no exchange of variation or initial margin, meaning that $C=I^{T C}=I^{F C}=0, d \mathbb{Q} \otimes d t$-a.s. Exposures on such transactions are to be funded by the internal treasury desk of the bank, hence, due to internal rules of the bank, the front office desk decides to discount cashflows via a synthetic unsecured discount curve with associated short rate process $r^{f}=\left(r_{t}^{f}\right)_{t \in[0, T]}$ defined via $r^{f}=\frac{r^{f, l}+r^{f, b}}{2}$.

Hence, the official clean price from the bank perspective is

$$
\hat{\mathcal{P}}_{t}^{1}=\mathbb{E}^{\mathbb{Q}}\left[B_{t}^{f} \int_{(t, T]} \frac{d A_{u}^{1}}{B_{u}^{f}} \mid \mathcal{F}_{t}\right]=B_{t}^{f} \mathbb{E}^{\mathbb{Q}}\left[\frac{S_{T}-K^{1}}{B_{T}^{f}} \mid \mathcal{F}_{t}\right] .
$$

The xVA desk is forced by the internal policy of the bank to employ (4.4) as the official clean price for the transaction. However, using Proposition 3.21 it is possible to compute a consistent price which is then given by

$$
\mathscr{V}_{t}^{1}(\varphi)=V_{t}^{1}(\varphi)=\hat{\mathcal{P}}_{t}^{1}-X V A_{t}^{1}-\operatorname{DiscV} A_{t}^{1}
$$

where

$$
\begin{aligned}
X V A_{t}^{1} & =-C V A_{t}^{1}+D V A_{t}^{1}+F V A_{t}^{1} \\
& =-B_{t}^{r} \mathbb{E}^{\mathbb{Q}}\left[1_{\left\{\tau^{C}<\tau^{B}\right\}}\left(1-R^{C}\right) \frac{1}{B_{\tau}^{r}}\left(\hat{\mathcal{P}}_{\tau}^{1}-D i s c V A_{\tau}^{1}\right)^{-} \mid \mathcal{G}_{t}\right], \\
& +B_{t}^{r} \mathbb{E}^{\mathbb{Q}}\left[1_{\left\{\tau^{B}<\tau^{C}\right\}}\left(1-R^{B}\right) \frac{1}{B_{\tau}^{r}}\left(\hat{\mathcal{P}}_{\tau}^{1}-D i s c V A_{\tau}^{1}\right)^{+} \mid \mathcal{G}_{t}\right], \\
& +B_{t}^{r} \mathbb{E}^{\mathbb{Q}}\left[\int_{t}^{\tau} \frac{\left(r_{u}^{f, l}-r_{u}\right)\left(\mathscr{V}_{u}^{1}(\varphi)\right)^{+}-\left(r_{u}^{f, b}-r_{u}\right)\left(\mathscr{V}_{u}^{1}(\varphi)\right)^{-}}{B_{u}^{r}} d u \mid \mathcal{G}_{t}\right],
\end{aligned}
$$

while the discounting adjustment is

$$
\operatorname{DiscV} A_{t}^{1}:=B_{t}^{f} \mathbb{E}^{\mathbb{Q}}\left[\int_{t}^{T}\left(r_{u}-r_{u}^{f}\right) \frac{\hat{V}_{u}^{1}}{B_{u}^{f}} d u \mid \mathcal{F}_{t}\right] .
$$


The $\mathbb{G}$-BSDE solved by 4.5 is given by

$$
\left\{\begin{aligned}
&-d X V A_{t}^{1}=-\left.f\left(t, \hat{V}^{1}-X V A^{1}, 0,0\right)+r_{t} X V A_{t}^{1}\right] d t \\
& \quad-\sum_{k=1}^{d} \tilde{Z}_{t}^{k} d W_{t}^{k, \mathbb{Q}}-\sum_{j \in\{B, C\}} \tilde{U}_{t}^{j} d M_{t}^{j, \mathbb{Q}} \\
& X V A_{\tau}^{1}=\hat{V}_{\tau}^{1}(\varphi)-\theta_{\tau}\left(\hat{V}^{1}, 0,0\right) .
\end{aligned}\right.
$$

We observe that the non-linearity effect $N L_{t}\left(V^{1}\right)=0$ is of course zero, since the portfolio between the bank and the counterparty consists of a single contingent claim.

Assume now that the counterparty is interested in a second product, e.g. a second forward contract on the risky asset $S$ with maturity $T$ and opposite direction, so that

$$
A_{t}^{2}=1_{\{t=T\}}\left(K^{2}-S_{T}\right) .
$$

In line with the previous reasoning, the clean values from the perspective of the xVA desk and the front-office desk are respectively

$$
\hat{V}_{t}^{2}(\varphi)=B_{t}^{r} \mathbb{E}^{\mathbb{Q}}\left[\frac{K^{2}-S_{T}}{B_{T}^{r}} \mid \mathcal{F}_{t}\right], \quad \hat{\mathcal{P}}_{t}^{2}=B_{t}^{r} \mathbb{E}^{\mathbb{Q}}\left[\frac{K^{2}-S_{T}}{B_{T}^{f}} \mid \mathcal{F}_{t}\right] .
$$

Given the presence of the first forward contract in the portfolio, the full value of the portfolio, now including the second claim, is

$$
\mathscr{V}_{t}^{2}(\varphi)=\hat{\mathcal{P}}_{t}^{1}+\hat{\mathcal{P}}_{t}^{2}-X V A_{t}^{2}-\operatorname{DiscV} A_{t}^{1}-\operatorname{DiscV} A_{t}^{2},
$$

where

$$
\begin{aligned}
X V A_{t}^{2} & =-C V A_{t}^{2}+D V A_{t}^{2}+F V A_{t}^{2} \\
& =-B_{t}^{r} \mathbb{E}^{\mathbb{Q}}\left[1_{\left\{\tau^{C}<\tau^{B}\right\}}\left(1-R^{C}\right) \frac{1}{B_{\tau}^{r}}\left(\hat{\mathcal{P}}_{\tau}^{1}+\hat{\mathcal{P}}_{\tau}^{2}-\operatorname{DiscV} A_{\tau}^{1}-D i s c V A_{\tau}^{2}\right)^{-} \mid \mathcal{G}_{t}\right], \\
& +B_{t}^{r} \mathbb{E}^{\mathbb{Q}}\left[1_{\left\{\tau^{B}<\tau^{C}\right\}}\left(1-R^{B}\right) \frac{1}{B_{\tau}^{r}}\left(\hat{\mathcal{P}}_{\tau}^{1}+\hat{\mathcal{P}}_{\tau}^{2}-D i s c V A_{\tau}^{1}-D i s c V A_{\tau}^{2}\right)^{+} \mid \mathcal{G}_{t}\right], \\
& +B_{t}^{r} \mathbb{E}^{\mathbb{Q}}\left[\int_{t}^{\tau} \frac{\left(r_{u}^{f, l}-r_{u}\right)\left(\mathscr{V}_{u}^{2}(\varphi)\right)^{+}-\left(r_{u}^{f, b}-r_{u}\right)\left(\mathscr{V}_{u}^{2}(\varphi)\right)^{-}}{B_{u}^{r}} d u \mid \mathcal{G}_{t}\right],
\end{aligned}
$$

and $\operatorname{DiscV} A^{2}$ is given by

$$
\operatorname{DiscV} A_{t}^{2}:=B_{t}^{f} \mathbb{E}^{\mathbb{Q}}\left[\int_{t}^{T}\left(r_{u}-r_{u}^{f}\right) \frac{\hat{V}_{u}^{2}}{B_{u}^{f}} d u \mid \mathcal{F}_{t}\right] .
$$

The solution to the $\mathbb{G}-\mathrm{BSDE}$

$$
\left\{\begin{aligned}
-d X V A_{t}^{2}=- & {\left[f\left(t, \hat{V}^{2}-X V A^{2}, 0,0\right)+r_{t} X V A_{t}^{2}\right] d t } \\
& -\sum_{k=1}^{d} \tilde{Z}_{t}^{k} d W_{t}^{k, \mathbb{Q}}-\sum_{j \in\{B, C\}} \tilde{U}_{t}^{j} d M_{t}^{j, \mathbb{Q}} \\
X V A_{\tau}^{2}= & \left(\hat{V}_{\tau}^{1}(\varphi)+\hat{V}_{\tau}^{2}(\varphi)-\theta_{\tau}\left(\hat{V}^{1}+\hat{V}^{2}, 0,0\right)\right)
\end{aligned}\right.
$$

is given by 4.5 .

Given the presence of the first claim in the portfolio, the $\mathrm{xVA}$ charge on the second claim is $\Delta X V A=$ 
$X V A^{2}-X V A^{1}$, whereas the non-linearity is effect is given by

$$
\begin{aligned}
N L_{t}\left(V^{2}\right) & =X V A_{t}^{2}+B_{t}^{r} \mathbb{E}^{\mathbb{Q}}\left[1_{\left\{\tau^{C}<\tau^{B}\right\}}\left(1-R^{C}\right) \frac{1}{B_{\tau}^{r}}\left(\hat{\mathcal{P}}_{\tau}^{2}-\operatorname{DiscV} A_{\tau}^{2}\right)^{-} \mid \mathcal{G}_{t}\right], \\
& -B_{t}^{r} \mathbb{E}^{\mathbb{Q}}\left[1_{\left\{\tau^{B}<\tau^{C}\right\}}\left(1-R^{B}\right) \frac{1}{B_{\tau}^{r}}\left(\hat{\mathcal{P}}_{\tau}^{2}-D i s c V A_{\tau}^{2}\right)^{+} \mid \mathcal{G}_{t}\right] \\
& -B_{t}^{r} \mathbb{E}^{\mathbb{Q}}\left[\int_{t}^{\tau} \frac{\left(r_{u}^{f, l}-r_{u}\right)\left(V_{u}^{2}(\varphi)\right)^{+}-\left(r_{u}^{f, b}-r_{u}\right)\left(V_{u}^{2}(\varphi)\right)^{-}}{B_{u}^{r}} d u \mid \mathcal{G}_{t}\right] .
\end{aligned}
$$

Observe that in the last FVA term appearing in 4.12 we have the presence of $V^{2}$, i.e. a portfolio consisting only of the second claim: all expectations in (4.12) represent the stand-alone xVA correction for the second contingent claim. In 4.10) we observe instead the presence of $\mathscr{V}^{2}$, i.e. a portfolio consisting of the first and the second claim. The role of netting effects in reducing the overall impact of value adjustments can be seen by observing that

$$
\begin{aligned}
& \hat{\mathcal{P}}_{t}^{1}+\hat{\mathcal{P}}_{t}^{2}-\left(\operatorname{DiscV} A_{t}^{1}+\operatorname{DiscV} A_{t}^{2}\right) \\
& =\left(B_{t}^{f} \mathbb{E}^{\mathbb{Q}}\left[\frac{1}{B_{T}^{f}} \mid \mathcal{F}_{t}\right]-B_{t}^{f} \mathbb{E}^{\mathbb{Q}}\left[\int_{t}^{T}\left(r_{u}-r_{u}^{f}\right) \frac{B_{u}^{r}}{B_{u}^{f}} \mathbb{E}^{\mathbb{Q}}\left[\frac{1}{B_{T}^{r}} \mid \mathcal{F}_{u}\right] d u \mid \mathcal{F}_{t}\right]\right)\left(K^{2}-K^{1}\right) \\
& =B^{r}(t, T)\left(K^{2}-K^{1}\right),
\end{aligned}
$$

where $B^{r}(t, T)$ denotes the time $t$ price of a zero coupon bond with maturity $T$ and short rate $r$. This shows that the combined exposure of the two forward contracts is obviously independent of the volatility of the asset $S$.

We finally stress that, given a numerical scheme that allows to estimate the evolution of the conditional expectation $\hat{V}$, e.g. a regression estimator in the context of a Monte Carlo simulation, the xVA desk can immediately estimate the DiscVA, hence only a simulation in terms of $r$ discounting is required for the implementation.

4.1. A numerical illustration. We conclude the paper by presenting two numerical illustrations 3 , The first one aims at providing evidence regarding the last claim of the previous section, namely that the estimation of DiscVA is a feasible task. We postulate that the risky asset evolves according to (4.1). We assume, for the sake of simplicity, that all parameters are constant and we set $r^{r}=r=0.01$, $\kappa=0, \sigma=0.25, S_{0}=100$. In line with the previous section we consider the forward contract 4.2 written on 1000 units of $S$ with strike $K^{1}=80$ and $T_{1}=1$.

For such a simple claim we can compute prices without resorting to simulations. In particular, the xVA desk computes the price $\hat{V}_{0}^{1}$ according to 4.3 , i.e. $\hat{V}_{0}^{1}=20795.22 \mathrm{EUR}$, obtained by assuming a perfect collateralization with collateral rate $r$. However, the claim is perfectly uncollateralized, hence the front office function employs for valuation the unsecured discount rate $r^{f}=0.05$ and hence computes the price $P_{0}^{1}$ according to (4.4). The front office price is $P_{0}^{1}=19980.62$ EUR. As we have already seen, the front office price is not consistent with the portfolio-wide valuation of the $\mathrm{xVA}$ desk, however the xVA desk can solve the consistency problem by computing $\operatorname{DiscV} A_{0}^{1}$ as in 4.6 .

In this very simple example $\operatorname{DiscV} A_{0}^{1}$ can be computed in closed form and we have $\operatorname{DiscV} A_{0}^{1}=$ -814.70 .

Note however that in more general cases the computation of $\operatorname{DiscV} A$ can be performed at no additional costs via Monte Carlo methods, which are usually employed for xVA simulations.

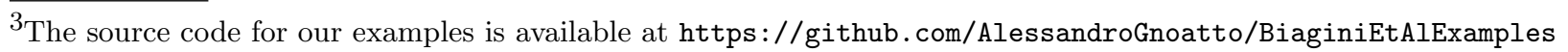


The second experiment we propose aims at showing the relevance of portfolio effects in xVA computations. Again a simple example will suffice to provide enough intuition. To illustrate the issue we simplify the treatment by assuming that there is a unique risk-free rate $r$ involved in all valuations. We consider again the bank trading two forwards on the stock $S$ as before. We assume again $T^{1}=1$ and set $K^{1}=S_{0}=100$. We suppose that the forward is written on 1000 units of the stock, and that only the counterparty can default. In summary, the whole xVA adjustment is solely given by the CVA. We suppose that the counterparty has a constant hazard rate $\lambda^{C, \mathbb{Q}}=0.04$ and a recovery rate $R^{C}=0.4$.

The base scenario we consider is represented by the sole presence of the forward above in the portfolio between the bank and the counterparty. Using the same Monte Carlo framework we developed for the previous numerical test, we simulate again paths of the underlying $S$. After that, we perform a pathwise simulation of the exposure of the forward, which we then numerically integrate with respect to time and average over all paths. This procedure produces a Monte Carlo estimation of the CVA under the filtration $\mathbb{F}$ according to Corollary 3.17. We obtain an estimate for $C V A_{0}^{1}=148.17$ EUR. Let us now introduce the second forward mentioned above, where we assume again that $T^{2}=T^{1}=1$ and set $K^{2}=90$. We also suppose that the second forward is written on the same quantity of shares of $S$, namely 1000. We observe that, due to the different strikes, the second forward does not perfectly offset the first one. We first assumed that the second forward is the only claim in the portfolio and thus obtained an estimate of the stand alone CVA of 309.22 EUR, so that the sum of the CVAs of the two forwards, ignoring portfolio effects, is 457.49 EUR. Such value clearly overestimates the outstanding credit exposure between the bank and the counterparty.

By relying on a Monte Carlo simulation under the same assumptions as above, we compute the portfolio-wide CVA, i.e. $C V A^{2}$ and we obtain the estimate $C V A^{2}=232.69$ EUR. We observe then that the incremental CVA, $\triangle C V A=84.52$ EUR. Finally, the non-linearity from (3.55) is estimated by $N L_{0}\left(V^{2}\right)=232.69-84.52=148.17$ EUR.

The example we propose shows quite clearly the relevance of portfolio effects: if the xVA desk ignored portfolio effects, the xVA charge would be 457.49 EUR. By applying the incremental approach to xVA charge instead, when the second forward is included in the portfolio, there is only an additional charge of 84.52 EUR. This is due to the fact that the two credit exposure partially compensate each other. In Figure 2 we provide a further visualization of the portfolio effects. We compute the Monte Carlo sample average of the negative and positive part of the credit exposure of the forwards under consideration: such quantities are usually termed expective negative (resp. positive) exposure. Also, we compute the $95 \%$-quantile of the exposure. Red lines correspond to the first forward with strike $K^{1}$ whereas green lines refer to the second forward with strike $K^{2}$. Finally, the portfolio resulting from the combination of the two forwards is represented by a blue line. We can clearly observe that combining the two claims has a beneficial effect in terms of reduction of the exposure: in particular we observe that the $95 \%$-quantile is constant.

\section{Appendix A. Existence and Uniqueness of BSDEs}

In this section we review some results on existence and uniqueness for some BSDEs. Our main references are Nie and Rutkowski (2016), which in turn extends results from Carbone et al. (2008), and Agarwal et al. (2018).

Let $M=\left(M^{1}, \ldots M^{d}\right)$ be a $d$-dimensional, real-valued, continuous and square integrable martingale on a filtered probability space $(\Omega, \mathcal{F}, \mathbb{F}, \mathbb{Q})$, where the filtration is assumed to satisfy the usual 

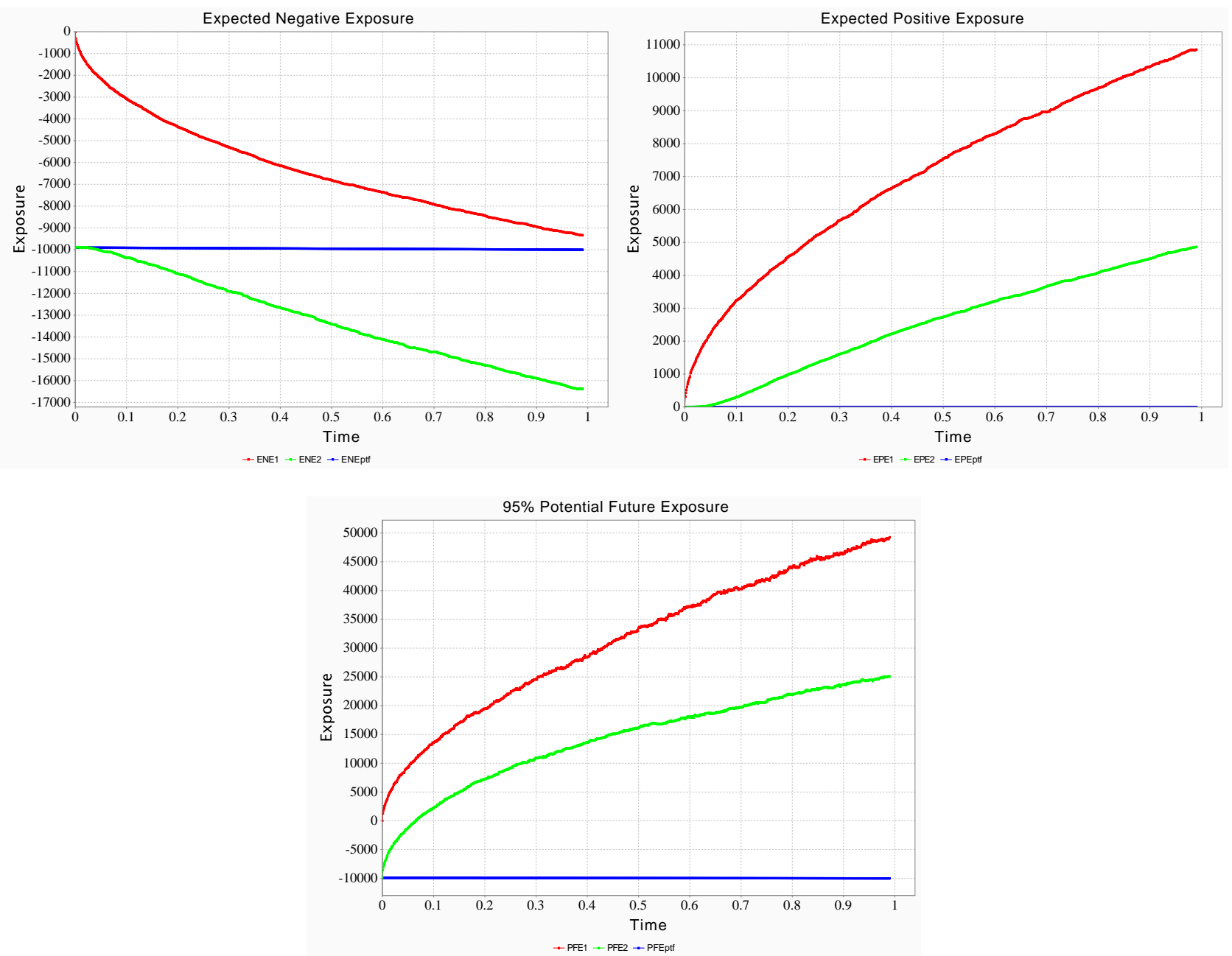

Figure 2. Expected positive and negative exposure and potential future exposure for the portfolio of two forwards. The red and green lines refer to the first and second forward taken in isolation, whereas the blu line represents a portfolio containing the two claims.

hypotheses and we assume that the predictable representation property holds with respect to $M$ for $(\mathbb{F}, \mathbb{Q})$-martingales. We use $\langle M\rangle$ to denote the quadratic variation of $M$.

Assumption A.1 Nie and Rutkowski (2016) Assumption 3.1). There exists an $\mathbb{R}^{d \times d}$-valued process $m$ and an $\mathbb{F}$-adapted, continuous, bounded, increasing process $Q$ with $Q_{0}=0$ such that, for all $t \in[0, T]$,

$$
\langle M\rangle_{t}=\int_{0}^{t} m_{u} m_{u}^{\top} d Q_{u}
$$

If $M=W$ is a one-dimensional standard Brownian motion, then $Q_{t}=t$, whereas $m$ corresponds to the identity matrix. Next we introduce the driver of the BSDE via the following

Assumption A.2 (Nie and Rutkowski (2016) Assumption 3.2). Let $h: \Omega \times[0, T] \times \mathbb{R} \times \mathbb{R}^{d} \mapsto \mathbb{R}$ be an $\mathcal{F} \otimes \mathcal{B}([0, T]) \otimes \mathcal{B}(\mathbb{R}) \otimes \mathcal{B}\left(\mathbb{R}^{d}\right)$-measurable function such that $h(\cdot, \cdot, y, z)$ is an $\mathbb{F}$-adapted process for any fixed $(y, z) \in \mathbb{R} \times \mathbb{R}^{d}$.

The BSDEs of interest in view of financial applications are forward-backward SDEs (FBSDEs). Following Nie and Rutkowski (2016), we introduce a generic (forward) factor matrix-valued process given 
by

$$
\mathbb{X}_{t}:=\left(\begin{array}{cccc}
X_{t}^{1} & 0 & \ldots & 0 \\
0 & X_{t}^{2} & \ldots & 0 \\
\vdots & \vdots & \ddots & 0 \\
0 & 0 & \ldots & X_{t}^{d}
\end{array}\right), t \in[0, T]
$$

where the auxiliary processes $X^{i}, i=1, \ldots, d$, are assumed to be $\mathbb{F}$-adapted. The processes $X^{i}$ represent market risk factors or traded assets. We assume that the function $h$ of Assumption A.2 can be written as $h(\omega, t, y, z)=g\left(\omega, t, y, \mathbb{X}_{t} z\right)$, for $g$ satisfying Assumption A.2.

Definition A.3 (Nie and Rutkowski (2016) Definition 4.1). We say that an $\mathbb{R}^{d \times d}$-valued process $\gamma$ satisfies the ellipticity condition if there exists a constant $\Lambda>0$ such that

$$
\sum_{i=1}^{d}\left(\gamma_{t} \gamma_{t}^{\top}\right)_{i j} a_{i} a_{j} \geq \Lambda\|a\|
$$

for all $a \in \mathbb{R}^{d}$ and $t \in[0, T]$.

Assumption A.4 Nie and Rutkowski 2016) Assumption 4.2). The $\mathbb{R}^{d \times d}$-valued $\mathbb{F}$-adapted process $m$ in (A.1) is given by

$$
m_{t} m_{t}^{\top}=\mathbb{X}_{t} \gamma_{t} \gamma_{t}^{\top} \mathbb{X}_{t}^{\top}
$$

where $\gamma=[\gamma]_{i j}$ is a d-dimensional square matrix of $\mathbb{F}$-adapted processes satisfying the ellipticity condition (A.2).

In the following we recall some definitions from Nie and Rutkowski (2016).

Definition A.5. We say that the function $h: \Omega \times[0, T] \times \mathbb{R} \times \mathbb{R}^{d} \mapsto \mathbb{R}$ satisfies

- the uniform Lipschitz condition if there exists a constant $L$ such that for any $t \in[0, T]$ and all $y_{1}, y_{2} \in \mathbb{R}, z_{1}, z_{2} \in \mathbb{R}^{d}$

$$
\left|h\left(t, y_{1}, z_{1}\right)-h\left(t, y_{2}, z_{2}\right)\right| \leq L\left(\left|y_{1}-y_{2}\right|+\left\|z_{1}-z_{2}\right\|\right) ;
$$

- the uniform $m$-Lipschitz condition if there exists a constant $\hat{L}$ such that for any $t \in[0, T]$ and all $y_{1}, y_{2} \in \mathbb{R}, z_{1}, z_{2} \in \mathbb{R}^{d}$

$$
\left|h\left(t, y_{1}, z_{1}\right)-h\left(t, y_{2}, z_{2}\right)\right| \leq \hat{L}\left(\left|y_{1}-y_{2}\right|+\left\|m_{t}^{\top}\left(z_{1}-z_{2}\right)\right\|\right) ;
$$

- the uniform $\mathbb{X}$-Lipschitz condition if there exists a constant $\tilde{L}$ such that for any $t \in[0, T]$ and all $y_{1}, y_{2} \in \mathbb{R}, z_{1}, z_{2} \in \mathbb{R}^{d}$

$$
\left|h\left(t, y_{1}, z_{1}\right)-h\left(t, y_{2}, z_{2}\right)\right| \leq \tilde{L}\left(\left|y_{1}-y_{2}\right|+\left\|\mathbb{X}_{t}\left(z_{1}-z_{2}\right)\right\|\right) .
$$

Lemma A.6 (Nie and Rutkowski (2016) Lemma 4.2). If Assumption A.4 holds and the generator $h$ is uniform $\mathbb{X}$-Lipschitz, then $h$ is uniform $m$-Lipschitz with $\hat{L}=\tilde{L} \max \left\{1, \Lambda^{-\frac{1}{2}}\right\}$, where $\Lambda$ is the constant defined in A.2.

Theorem A.7 provides the existence and uniqueness result, which is relevant for our purposes.

Theorem A.7 (Nie and Rutkowski (2016) Theorem 4.1). Assume that the function $h$ can be represented as $h(t, y, z)=g\left(t, y, \mathbb{X}_{t} z\right)$, where the function $g: \Omega \times[0, T] \times \mathbb{R} \times \mathbb{R}^{d} \mapsto \mathbb{R}$ satisfies the uniform Lipschitz condition. Let the process $h(\cdot, 0,0)$ belong to the space $\mathcal{H}^{2}(\mathbb{Q})$, the random variable $\eta$ belong 
to $L^{2}\left(\mathcal{F}_{T}, \mathbb{Q}\right)$ and $U$ be a real-valued $\mathbb{F}$-adapted process such that $U \in \mathcal{H}^{2}(\mathbb{Q})$ and $U_{T} \in L^{2}\left(\mathcal{F}_{T}, \mathbb{Q}\right)$. Assume that the process $m$ satisfies Assumption A.4 for some constant $\Lambda>0$. Then the BSDE

$$
\begin{cases}d Y_{t} & =Z_{t}^{\top} d M_{t}-h\left(t, Y_{t}, Z_{t}\right) d Q_{t}+d U_{t} \\ Y_{T} & =\eta\end{cases}
$$

has a unique solution $(Y, Z)$ such that $\left(Y, m^{\top} Z\right) \in \mathcal{H}^{2}(\mathbb{Q}) \times \mathcal{H}^{2, d}(\mathbb{Q})$. Moreover the processes $Y$ and U satisfy

$$
\mathbb{E}^{\mathbb{Q}}\left[\sup _{t \in[0, T]}\left|Y_{t}-U_{t}\right|^{2}\right]<\infty
$$

Assumption A.8 Agarwal et al. (2018) Assumption (S)). For any $y, z, \lambda \in \mathbb{R} \times \mathbb{R}^{d} \times \mathbb{R}, f(\cdot, y, z, \lambda)$ is an $\mathbb{F}$-adapted stochastic process with values in $\mathbb{R}$ and there exists a constant $C_{f}>0$ such that $\mathbb{P}$-a.s., for all $\left(s, y_{1}, z_{1}, \lambda_{1}\right),\left(s, y_{2}, z_{2}, \lambda_{2}\right) \in[0, T] \times \mathbb{R} \times \mathbb{R}^{d} \times \mathbb{R}$,

$$
\left|f\left(s, y_{1}, z_{1}, \lambda_{1}\right)-f\left(s, y_{2}, z_{2}, \lambda_{2}\right)\right| \leq C_{f}\left(\left|y_{1}-y_{2}\right|+\left|z_{1}-z_{2}\right|+\left|\lambda_{1}-\lambda_{2}\right|\right) .
$$

Moreover, $\mathbb{E}\left[\int_{0}^{T}|f(s, 0,0,0)|^{2} d s\right]<\infty$.

A.1. Proof of Proposition 2.11. Since we are only trading in the basic risky assets, the position in the initial margin is zero hence, by $(2.9)$ and $(2.17)$, the value process is of the form

$$
V_{t}(\varphi)=\psi_{t}^{f, b} B_{t}^{f, b}+\psi_{t}^{f, l} B_{t}^{f, l}, t \in[0, T] .
$$

Recalling that simultaneous borrowing and lending at the same time is not allowed, we have by A.4 that

$$
\psi^{f, l}=\left(V_{t}(\varphi)\right)^{+}\left(B_{t}^{f, l}\right)^{-1}, \psi^{f, b}=-\left(V_{t}(\varphi)\right)^{-}\left(B_{t}^{f, b}\right)^{-1}, t \in[0, T] .
$$

Moreover, we can rewrite the funding term of the generic $i$-th risky assets as follows

$$
\int_{0}^{t} \psi_{u}^{i} d B_{u}^{i}=-\int_{0}^{t} \frac{\xi_{u}^{i} S_{u}^{i}}{B_{u}^{i}} d B_{u}^{i}=-\int_{0}^{t} r_{u}^{i} \xi_{u}^{i} S_{u}^{i} d u, t \in[0, T] .
$$

Upon substitution in the self-financing condition (2.18), we obtain

$d V_{t}(\varphi)=\sum_{i=1}^{d} \xi_{t}^{i}\left(d S_{t}^{i}+d D_{t}^{i}-r_{t}^{i} S_{t}^{i} d t\right)+\sum_{j \in\{B, C\}} \xi_{t}^{j}\left(d P_{t}^{j}-r_{t}^{j} P_{t-}^{j} d t\right)-r_{t}^{f, b}\left(V_{t}(\varphi)\right)^{-} d t+r_{t}^{f, l}\left(V_{t}(\varphi)\right)^{+} d t$.

We now use the inequality $r_{t}^{f, l} \leq r_{t}^{f, b}$ from Assumption 2.10, hence

$$
\begin{aligned}
d V_{t}(\varphi) & =\sum_{i=1}^{d} \xi_{t}^{i}\left(d S_{t}^{i}+d D_{t}^{i}-r_{t}^{i} S_{t}^{i} d t\right)+\sum_{j \in\{B, C\}} \xi_{t}^{j}\left(d P_{t}^{j}-r_{t}^{j} P_{t-}^{j} d t\right)+r_{t}^{f, l}\left(V_{t}(\varphi)\right)^{+} d t-r_{t}^{f, b}\left(V_{t}(\varphi)\right)^{-} d t \\
& \leq \sum_{i=1}^{d} \xi_{t}^{i}\left(d S_{t}^{i}+d D_{t}^{i}-r_{t}^{i} S_{t}^{i} d t\right)+\sum_{j \in\{B, C\}} \xi_{t}^{j}\left(d P_{t}^{j}-r_{t}^{j} P_{t-}^{j} d t\right)+r_{t}^{f, l} V_{t}(\varphi) d t .
\end{aligned}
$$

Introducing $\tilde{V}_{t}^{l}(\varphi):=\left(B_{t}^{f, l}\right)^{-1} V_{t}(\varphi)$, we have then the inequality

$$
d \tilde{V}_{t}^{l}(\varphi) \leq \sum_{i=1}^{d} \xi_{t}^{i}\left(B_{t}^{f, l}\right)^{-1}\left(d S_{t}^{i}+d D_{t}^{i}-r_{t}^{i} S_{t}^{i} d t\right)+\sum_{j \in\{B, C\}} \xi_{t}^{j}\left(B_{t}^{f, l}\right)^{-1}\left(d P_{t}^{j}-r_{t}^{j} P_{t-}^{j} d t\right)
$$


or equivalently,

$$
d \tilde{V}_{t}^{l}(\varphi) \leq \sum_{i=1}^{d} \xi_{t}^{i} \frac{B_{t}^{i}}{B_{t}^{f, l}} \frac{\left(d S_{t}^{i}+d D_{t}^{i}-r_{t}^{i} S_{t}^{i} d t\right)}{B_{t}^{i}}+\sum_{j \in\{B, C\}} \xi_{t}^{j} \frac{B_{t}^{j}}{B_{t}^{f, l}} \frac{\left(d P_{t}^{j}-r_{t}^{j} P_{t-}^{j} d t\right)}{B_{t}^{j}},
$$

and so, by 2.19), we arrive at the inequality

$$
d \tilde{V}_{t}^{l}(\varphi) \leq \sum_{i=1}^{d} \xi_{t}^{i} \frac{B_{t}^{i}}{B_{t}^{f, l}} d \tilde{S}_{t}^{i, c l d}+\sum_{j \in\{B, C\}} \xi_{t}^{j} \frac{B_{t}^{j}}{B_{t}^{f, l}} d \tilde{P}_{t}^{j},
$$

We observe that the right-hand side in A.5 is a local martingale, which is bounded from below by Definition 2.7 and because $r^{f}$ is bounded. This implies that the aforementioned right-hand side is a supermartingale. Absence of arbitrage follows along the usual lines.

\section{ACKNOWLEDGMENTS}

We would like to thank the reviewers for their useful remarks that helped us improve our work. We are also grateful to Damiano Brigo, Agostino Capponi, Andrea Pallavicini, and the participants to the SIAM Conference on Financial Mathematics \& Engineering in Toronto, the XX Quantitative Finance Workshop in Zurich, and the Mathematical and Computational Finance Internal Seminar, Oxford University, for their comments and suggestions. Any errors remain our responsibility.

\section{REFERENCES}

Agarwal, A., De Marco, S., Gobet, E., López-Salas, J., Noubiagain, F., and Zhou, A. (2018). Backward stochastic differential equations arising in initial margin requirements. Preprint hal-01686952v2.

Aksamit, A. and Fontana, C. (2019). Martingale spaces and representations under absolutely continuous changes of probability. Electronic Communications in Probability, 24(none):1 - 13.

Albanese, C., Caenazzo, S., and Crépey, S. (2016). Capital Valuation Adjustment and Funding Valuation Adjustment. ArXiv e-prints, page arXiv:1603.03012.

Albanese, C., Caenazzo, S., and Crépey, S. (2017). Credit, funding, margin, and capital valuation adjustments for bilateral portfolios. Probability, Uncertainty and Quantitative Risk, 2(1):7.

Albanese, C. and Crépey, S. (2017). XVA Analysis From the Balance Sheet. Preprint.

Andersen, L., Duffie, D., and Song, Y. (2019). Funding value adjustments. The Journal of Finance, 74(1):145-192.

Ballotta, L., Fusai, G., and Marazzina, D. (2019). Integrated structural approach to credit value adjustment. European Journal of Operational Research, 272(3):1143 - 1157.

BCBS (2014). The standardized approach for measuring counterparty credit risk exposures. Technical Report 279. Basel Committee Paper.

Bichuch, M., Capponi, A., and Sturm, S. (2018a). Arbitrage-free XVA. Mathematical Finance, $28(2): 582-620$.

Bichuch, M., Capponi, A., and Sturm, S. (2018b). Robust XVA. arXiv e-prints, page arXiv:1808.04908.

Bielecki, T. and Rutkowski, M. (2004). Credit Risk: Modeling, Valuation, and Hedging. Financial Engineering Explained. Springer Finance.

Bielecki, T. and Rutkowski, M. (2015). Valuation and hedging of contracts with funding costs and collateralization. SIAM J. Finan. Math., 6(1):594-655.

Bielecki, T. R., Cialenco, I., and Rutkowski, M. (2018). Arbitrage-free pricing of derivatives in nonlinear market models. Probability, Uncertainty and Quantitative Risk, 3(1):2. 
Brigo, D., Buescu, C., Francischello, M., Pallavicini, A., and Rutkowski, M. (2018). Risk-neutral valuation under differential funding costs, defaults and collateralization. ArXiv e-prints.

Brigo, D., Capponi, A., and Pallavicini, A. (2014). Arbitrage-free bilateral counterparty risk valuation under collateralization and application to credit default swaps. Mathematical Finance, 24(1):125146.

Brigo, D., Francischello, M., and Pallavicini, A. (2019). Nonlinear valuation under credit, funding and margins: existence uniqueness, invariance and disentanglement. European Journal of Operational Research, 274(2):788-805.

Brigo, D. and Masetti, M. (2005). Risk neutral pricing of counterparty risk. In Pykhtin, M., editor, Counterparty Credit Risk Modeling: Risk Management, Pricing and Regulation. Risk Books, London.

Brigo, D. and Morini, M. (2018). Dangers of bilateral counterparty risk: the fundamental impact of closeout conventions. http://arxiv.org/abs/1011.3355v1.

Brigo, D., Morini, M., and Pallavicini, A. (2013). Counterparty Credit Risk, Collateral and Funding. Wiley Finance. Wiley, first edition.

Brigo, D. and Pallavicini, A. (2014). Nonlinear consistent valuation of ccp cleared or csa bilateral trades with initial margins under credit, funding and wrong-way risks. Journal of Financial Engineering, 01(01):1450001.

Brigo, D., Pallavicini, A., and Papatheodorou, V. (2011). Arbitrage-free valuation of bilateral counterparty risk for interest-rate products: Impact of volatilities and correlations. International Journal of Theoretical and Applied Finance, 14(06):773-802.

Burgard, C. and Kjaer, M. (2011a). In the balance. Risk, November:72-75.

Burgard, C. and Kjaer, M. (2011b). Partial differential equation representations of derivatives with bilateral counterparty risk and funding costs. The Journal of Credit Risk, 7(3):1-19.

Burgard, C. and Kjaer, M. (2013). Funding costs, funding strategies. Risk, Dec 2013:82-87.

Carbone, R., Ferrario, B., and Santacroce, M. (2008). Backward stochastic differential equations driven by càdlàg martingales. Theory Probab. Appl., 52(2):304-314.

Castagna, A. (2013). Funding valuation adjustment (fva) and theory of the firm: A theoretical justification of the inclusion of funding costs in the evaluation of financial contracts. Available at http://papers.ssrn.com/sol3/papers. cfm?abstract_id=2278595.

Castagna, A. (2014). Towards a theory of internal valuation and transfer pricing of products in a bank: Funding, credit risk and economic capital. Available at http://papers.ssrn.com/sol3/ papers.cfm?abstract_id=2392772.

Cherubini, U. (2005). Counterparty risk in derivatives and collateral policies: The replicating portfolio approach. In Tilman, L., editor, ALM of Financial Institutions. Institutional Investor Books.

Crépey, S. (2015a). Bilateral counterparty risk under funding constraints - part i: Pricing. Mathematical Finance, 25(1):1-22.

Crépey, S. (2015b). Bilateral counterparty risk under funding constraints - part ii: Cva. Mathematical Finance, 25(1):23-50.

Crépey, S., Bielecki, T. S., and Brigo, D. (2014). Counterparty risk and funding: a tale of two puzzles, volume 31 of Chapman and Hall/CRC Press Series in Financial Mathematics. Chapman and Hall/CRC, first edition.

Crépey, S., Sabbagh, W., and Song, S. (2020). When capital is a funding source: The anticipated backward stochastic differential equations of x-value adjustments. SIAM Journal on Financial Mathematics, 11(1):99-130. 
Duffie, D. and Huang, M. (1996). Swap rates and credit quality. The Journal of Finance, 51(3):921949.

El Karoui, N., Peng, S., and Quenez, M. C. (1997). Backward stochastic differential equations in finance. Mathematical Finance, 7(1):1-71.

Fujii, M., Shimada, A., and Takahashi, A. (2010). Note on construction of multiple swap curves with and without collateral. Preprint (available at http://ssrn.com/abstract=1440633).

Fujii, M., Shimada, A., and Takahashi, A. (2011). A market model of interest rates with dynamic basis spreads in the presence of collateral and multiple currencies. Wilmott, 54:61-73.

Garcia Trillos, C. A., Henrard, M. P. A., and Macrina, A. (2016). Estimation of future initial margins in a multi-curve interest rate framework. SSRN eLibrary.

Gnoatto, A. and Seiffert, N. (2020). Cross Currency Valuation and Hedging in the Multiple Curve Framework. arXiv e-prints, page arXiv:2001.11012.

Green, A. (2015). XVA: Credit, Funding and Capital Valuation Adjustments. Wiley Finance. John Wiley and Sons Inc, Chichester.

Gregory, J. (2015). The xVA challenge. Wiley Finance. Wiley, third edition.

Hull, J. and White, A. (2012). The fva debate. Risk, 25(July):83-85.

ISDA (2018). Isda simm methodology, version 2.1. Technical report.

Lichters, R., Stamm, R., and Gallagher, D. (2015). Modern Derivatives Pricing and Credit Exposure Analysis: Theory and Practice of CSA and XVA Pricing, Exposure Simulation and Backtesting. Applied Quantitative Finance. Palgrave Macmillan, first edition.

Mackenzie Smith, R. (2017). Banks calm on eurex-lch basis volatility.

Nie, T. and Rutkowski, M. (2016). Bsdes driven by multidimensional martingales and their applications to markets with funding costs. Theory Probab. Appl., 60(4):604-630.

Pallavicini, A., Perini, D., and Brigo, D. (2011). Funding Valuation Adjustment: a consistent framework including CVA, DVA, collateral,netting rules and re-hypothecation. ArXiv e-prints, page arXiv:1112.1521.

Piterbarg, V. (2010). Funding beyond discounting: collateral agreements and derivatives pricing. Risk Magazine, 2:97-102.

Piterbarg, V. (2012). Cooking with collateral. Risk Magazine, 2:58-63.

Sokol, A. (2014). Long-Term Portfolio Simulation: For XVA, Limits, Liquidity and Regulatory Capital. Risk Books, London.

(Francesca Biagini) LMU München, Mathematics Institute,

Theresienstr. 39, D-80333 Munich, Germany

Email address, Francesca Biagini: biagini@math.lmu.de

(Alessandro Gnoatto) University of Verona, Department of Economics,

via Cantarane 24, 37129 Verona, Italy

Email address, Alessandro Gnoatto: alessandro.gnoatto@univr.it

(Immacolata Oliva) Sapienza University of Rome, Department of Methods and Models for Economics, TERRITORY AND FinANCE

Via del Castro Laurenziano 9, 00161 Rome, Italy

Email address, Immacolata Oliva: immacolata.oliva@uniroma1.it 